\title{
High-resolution imaging of bacterial spatial organization with Vertical Cell Imaging by Nanostructured Immobilisation (VerCINI)
}

Kevin D. Whitley ${ }^{1,2^{*}}$, Stuart Middlemiss ${ }^{1}$, Calum Jukes ${ }^{1}$, Cees Dekker ${ }^{2}$, Séamus Holden ${ }^{1 *}$

${ }^{1}$ Centre for Bacterial Cell Biology, Newcastle University, UK; ${ }^{2}$ Department of Bionanoscience, Kavli Institute of Nanoscience Delft, Delft University of Technology, The Netherlands

*Corresponding authors: kevin.whitlev@ncl.ac.uk, seamus.holden@ncl.ac.uk

\begin{abstract}
Light microscopy is indispensable for analysis of bacterial spatial organization. However, imaging in bacteria is difficult due to their small sizes and the fact that most species are non-spherical, meaning they typically lie horizontally on a microscope coverslip. This is especially problematic when considering that many essential bacterial processes-such as cell division-occur along the short axes of these cells, and so are viewed side-on by standard microscopy. We recently developed a pair of methods to overcome this problem by forcing cells to stand vertically during imaging, named VerCINI (Vertical Cell Imaging by Nanostructured Immobilisation) and $\mu$ VerCINI (Microfluidic VerCINI). The concept behind both methods is that cells are imaged while confined vertically inside cell traps made from a nanofabricated mould. By doing so, the short axes of the cells are rotated parallel to the microscope imaging plane and are imaged with high resolution. $\mu$ VerCINI combines the vertical cell confinement with microfluidics so that vertical imaging can be done during fluid exchange, such as during antibiotic perturbations. Here, we provide a practical guide to implementing both VerCINI and $\mu \mathrm{VerCINI}$, with detailed protocols and experience-based tips so that interested researchers can easily use one or both imaging methods to complement their current approaches.
\end{abstract}

\section{Introduction}

Although long underappreciated as amorphous sacks of enzymes, it is now clear that bacterial cells are highly spatially organized. How proteins dynamically organize and remodel large cellular structures such as the cell wall or the chromosome is a central question in bacterial cell biology. However, imaging cellular spatial organization and dynamics inside bacteria is challenging for several reasons. Firstly, bacteria are very small, with a typical diameter of $1 \mu \mathrm{m}$, not much larger than the $\sim 250 \mathrm{~nm}$ diffraction limit of visible light. Secondly, most bacteria are not spherically symmetric, but they exist in a wide range of shapes including rods and ovoids. Such bacteria typically lie horizontally on a microscope coverslip-with their long axes parallel to the imaging plane and their short axes orthogonal to the imaging plane (Figure 1a, top). Imaging structure and dynamics along the short axes is therefore difficult, both due to a significant amount of background signal from out-of-focus light and the fact that axial resolution is generally lower than lateral resolution (approximately $250 \mathrm{~nm}$ lateral vs $550 \mathrm{~nm}$ axial resolution ${ }^{1}$.

This is especially problematic when one considers that many core bacterial processes occur, or are organized, along these shorter axes. For example, the peptidoglycan cell wall is primarily synthesized circumferentially around the cell in many bacteria ${ }^{2-4}$. Similarly, when cells divide, their division machinery moves circumferentially around the cell septum to progressively synthesize the cell septum inwards ${ }^{5,6}$. When cells have divided, what was once the division plane becomes two daughter cell poles, which then also exist along the short axes of the cells. A large number of proteins localize 
a
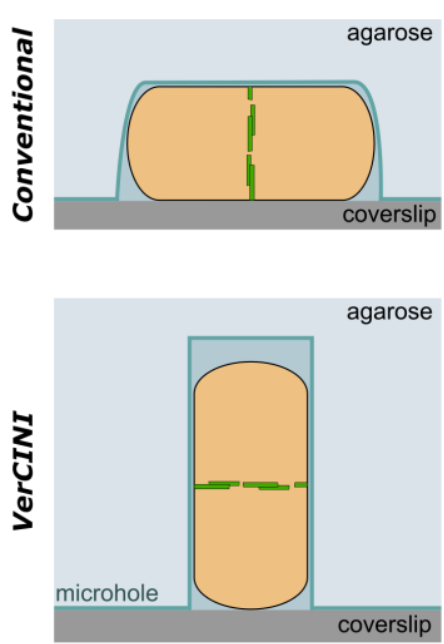

b
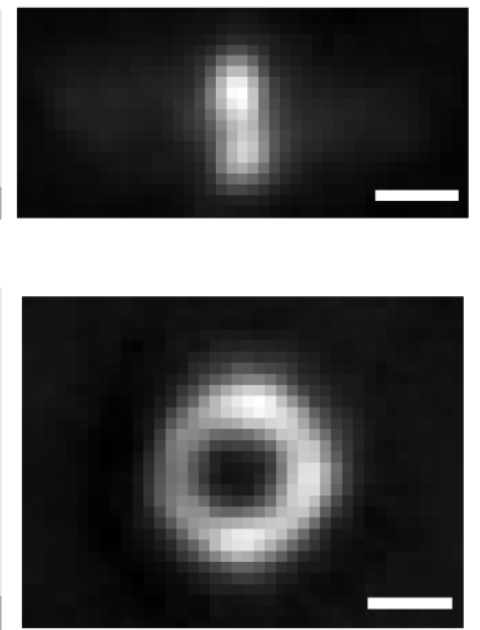
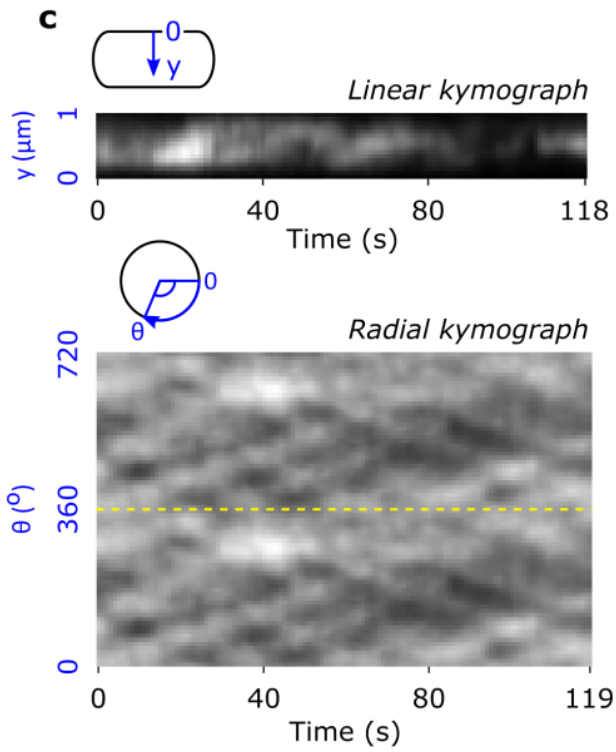

Figure 1: Concept of VerCINI and comparison to conventional imaging of division protein dynamics. (a) Schematics comparing conventional imaging and VerCINI to image division protein dynamics in rod-shaped cells. Division rings depicted as green dashed lines. Top: A cell lying horizontally under an agarose pad, with its division ring orthogonal to the microscope coverslip. Bottom: A cell confined vertically in an agarose microhole, with its division ring parallel to the microscope coverslip. (b) Representative images of $B$. subtilis cells expressing labelled FtsZ. Top: TIRF illumination of a cell expressing mNeonGreen-FtsZ ectopically from an inducible promoter (strain bWM $4^{28}$ ). The bottom of the division ring appears as a line across mid-cell. Bottom: HiLO illumination of a cell expressing FtsZ-GFP as a sole copy of FtsZ from the native locus (strain $\mathrm{SH} 130^{12}$ ). The full division ring appears as a circle. Scale bars: $500 \mathrm{~nm}$. (c) Kymographs of FtsZ treadmilling dynamics from cells in (b). Top: Kymograph of mNeonGreen-FtsZ treadmilling dynamics from 0-1 $\mu \mathrm{m}$ across the short axis of the cell. Diagonal lines show directional motion across the short axis of the cell. Bottom: A kymograph of FtsZ-GFP treadmilling dynamics around the cell circumference. Two full revolutions around the cell $\left(0-720^{\circ}\right)$ are plotted side-by-side to resolve filament trajectories that cross $0^{\circ} / 360^{\circ}$, separated by a yellow dotted line. Diagonal lines show directional motion around the full circumference of the cell. Raw data from Whitley, Jukes et al. $(2021)^{12}$.

specifically to the cell poles in rod-shaped bacteria that carry out a wide variety of functions, such as chemotaxis, motility, adhesion, virulence, chromosome organization, cell-cycle regulation, and secretion $^{7-11}$. A wealth of information on all these systems has been obtained from microscopy in recent years, but a major limitation remains that the horizontal orientation of cells is far from optimal for imaging these systems.

We recently developed two methods that enable high resolution imaging of bacteria along their short axes by orienting them vertically in nanofabricated cell traps ${ }^{5,12,13}$. In the first method, termed Vertical Cell Imaging by Nanostructured Immobilisation (VerCINI), cells are confined in 'microholes' formed in an agarose pad such that the short axes of the cell are in the microscope imaging plane (Figures 1a and $2 \mathrm{a}$, bottom). In the second method, termed Microfluidic VerCINI ( $\mu$ VerCINI), the microholes are opentopped, and the vertically trapped cells are confined within a microfluidic chamber to enable rapid solution exchange and chemical perturbation (Figure 3a). Both methods were originally developed to trap rod-shaped bacterial cells, which include the majority of model systems (e.g. Bacillus subtilis and Escherichia coli) and many human pathogens (e.g. Mycobacterium tuberculosis). However, they are suitable for any cells with cylindrical symmetry, which also includes ovococcal species such as the human pathogen Steptococcus pneumoniae.

Here we provide a detailed practical guide to implementing both VerCINI and $\mu$ VerCINI. We first give a general overview of the VerCINI method and its applications. We provide detailed workflows for all 
a
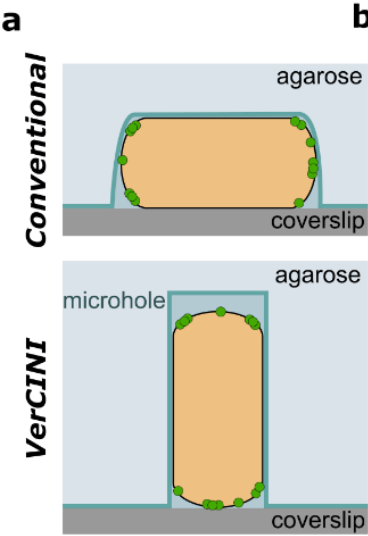

b
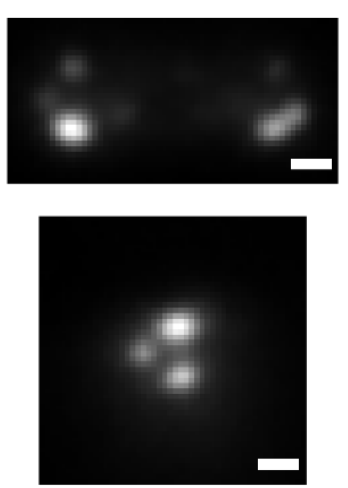

C

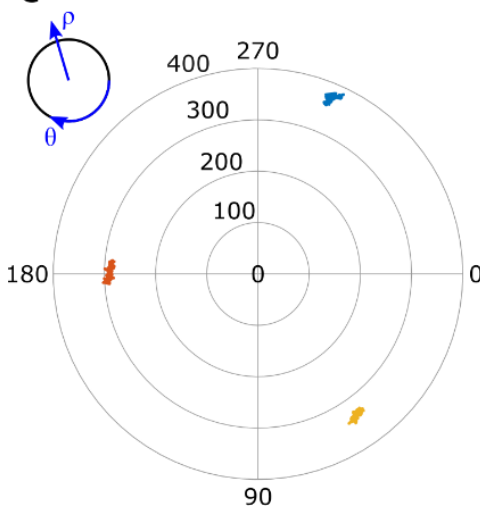

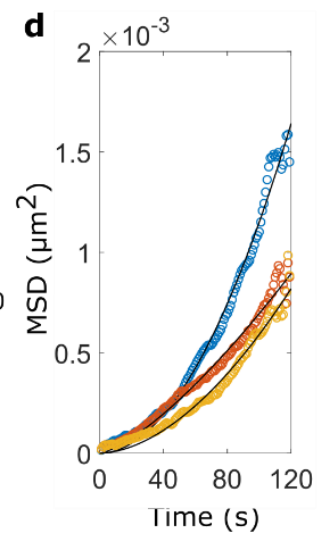

Figure 2: Concept of VerCINI and demonstration to conventional imaging of polar protein dynamics. (a) Schematics comparing conventional imaging and VerCINI to image polar proteins in rod-shaped cells. Polar proteins are depicted as green circles. Top: A cell lying horizontally under an agarose pad, with its poles orthogonal to the microscope coverslip. Bottom: A cell confined vertically in an agarose microhole, with its poles parallel to the microscope coverslip. (b) Representative images of $B$. subtilis cells expressing TlpA-mGFP ectopically from an inducible promoter (strain $\mathrm{HS}^{2} 8^{18}$ ). Top: Using conventional imaging with HiLO illumination the proteins appear as unresolved blobs at cell poles. Bottom: Using VerCINI with TIRF illumination, the proteins appear in several discrete clusters. Scale bars: $500 \mathrm{~nm}$. (c) Polar plot showing the motion of TlpA-mGFP clusters from the VerCINI imaging in panel (b). Motion of clusters tracked using TrackMate ${ }^{29}$. (d) Mean squared displacements of clusters in (c) for different time intervals (circles; colours correspond to those in (c)) with fits to generalized diffusion equation $\left\langle r^{2}(t)\right\rangle=K_{\alpha} t^{\alpha}$ (black lines).

aspects of VerCINI and $\mu \mathrm{VerCINI}$, from nanofabrication of micropillar arrays to acquisition and analysis of microscopy data. We provide rationales for the procedures described and include tips from our own experience in developing and using the methods to assist interested researchers. We also provide quantitative analysis of critical steps in the method to support troubleshooting and to enable future extensions and adaptations of the technique to new applications. By following the procedures described, this article should provide all information required for interested researchers to easily apply VerCINI to their own research.

\section{Principle and applications of VerCINI}

The key principle behind VerCINI is to rotate the short axes of bacteria into the microscope image plane by trapping cells in microholes formed from either agarose or PDMS (Figures 1a, 2a, and 3a). VerCINI offers several benefits for imaging the short axes of cells compared to conventional imaging of horizontally oriented cells:

- High resolution. A slice across the plane with the short axes of the cell (e.g. the cell septum) can be viewed at high $250 \mathrm{~nm}$ resolution (Figure 1 bottom), rather than just a thin volume of the bottom of the cell (TIRF microscopy, Figure 1 top) or low $550 \mathrm{~nm}$ resolution imaging via 3D fluorescence microscopy.

- Simultaneous imaging of an entire slice of the cell short-axes plane. An entire slice of the plane with the short axes of the cell can be imaged at once. Proteins that are primarily moving circumferentially, e.g. the divisome or elongasome proteins, can then be tracked for extended periods ${ }^{12}$, unlike TIRF, which truncates protein trajectories due to its small illumination volume. 
- High signal-to-noise ratio (SNR) imaging of the cell poles. By rotating cells vertically, the cell pole is placed in contact with/near to the microscope coverslip, and is also oriented to the microscope image plane. This allows high SNR imaging of the cell poles via TIRF illumination, while at the same time improving spatial resolution. Below, we demonstrate proof of concept application of VerCINI to imaging of cell pole protein dynamics (Figure 2).

In order to trap cells vertically, a silicon micropillar array serving as a negative master of the microholes must be fabricated at sufficient resolution for precise and reproducible cell trapping. In standard VerCINI, this micropillar array is used as a mould to form a microhole array in agarose. Bacteria are then immobilized within the traps, imaged using a high-resolution inverted microscope, and data analysed using custom image processing software tailored to maximise SNR of VerCINI microscopy data. Microfluidic VerCINI uses the same principle as standard VerCINI, but additionally enables rapid drug treatment of trapped cells by immobilizing bacteria in open-topped PDMS cell traps. VerCINI can also be combined with denoising, super-resolution microscopy, or single-particle tracking methods to improve image quality or resolution.

A key application for VerCINI is imaging the circumferential dynamics of cell division proteins. The septal peptidoglycan synthesis machinery moves circumferentially around the cell to build the cell wall, guided by the essential cytoskeletal protein $\mathrm{Fts}^{5,12}$. With conventional imaging, the division plane is orthogonal to the microscope imaging plane (Figure 1a, top), and hence the dynamics of division proteins are difficult to observe due the background signal from out-of-focus light coming from the rest of the division ring. This background signal can be largely removed using total internal reflection fluorescence (TIRF) illumination, which produces an evanescent wave that excites only the bottom 100-200 nm of the cell, but this limits imaging to only a small slice of the division septum (Figure 1b-c, top). In contrast, VerCINI allows simultaneous imaging of the entire septum, and strongly reduces background signal, because the septum no longer overlaps itself axially.

Using an early prototype of VerCINI, we observed the circumferential dynamics of FtsZ of the model rod-shaped Bacillus subtilis around the full division ring at high resolution and discovered that FtsZ filaments treadmill in the living cell (Figure $1 b-c$, bottom) ${ }^{5}$. VerCINI has since found a variety of applications in cell division microscopy. Using an optimized version of VerCINI with increased SNR, we were able to image FtsZ dynamics at near-single-filament resolution throughout the $B$. subtilis division cycle, demonstrating that FtsZ filament condensation into a dense Z-ring drives a transition in FtsZ dynamics from a mixed population of mobile and immobile filaments to stable treadmilling during constriction initiation and active septum building. With $\mu$ VerCINI we were also able to demonstrate that the FtsZ-targeting antibiotic PC190723 totally arrests FtsZ filament motion within seconds (Figure 3b-c). Beyond this, VerCINI has been applied to image division in several different bacterial species using a variety of imaging methods: The dynamics of FtsZ treadmilling in the ovococcoid bacterial pathogen Streptococcus pneumoniae ${ }^{13}$, the organization of FtsZ and FtsN in Escherichia coli using STED super-resolution imaging ${ }^{14}$, the division-associated cell wall synthase Ftsl in E. coli using single-particle tracking ${ }^{15}$, and peptidoglycan synthesis during division and elongation in S. pneumoniae using dSTORM with fluorescently-labelled 'clickable' D-amino acids ${ }^{16}$.

VerCINI also has multiple applications to cellular systems beyond cell division. Since the cell wall is synthesized circumferentially in many bacteria, nearly any protein involved in these processes is an ideal candidate for investigation with VerCINI. For example, we are currently using single-particle tracking to image the circumferential motion of the elongation-associated cytoskeletal protein MreB in B. subtilis (unpublished). Another possible application of VerCINI is the bacterial nucleoid and associated proteins, which may show significant radial organization due to the combined effects of transcription and translation ${ }^{17}$. 

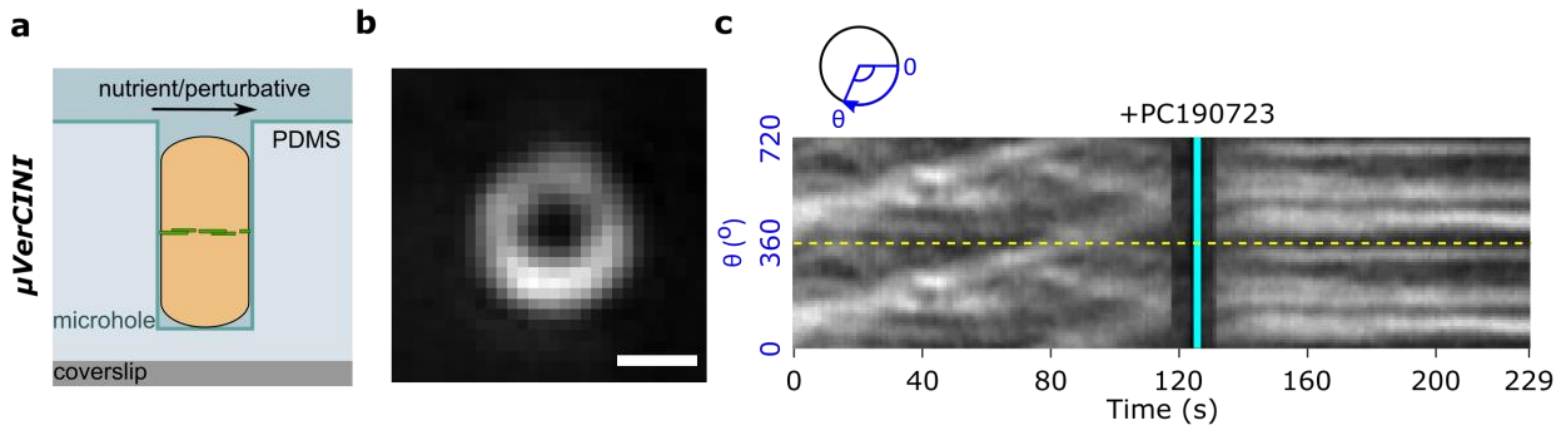

Figure 3: Concept of $\mu$ VerCINI and demonstration of imaging division protein dynamics during rapid antibiotic perturbation. (a) Schematic depicting a cell confined vertically in an open-topped PDMS microhole inside a microfluidic chamber, with its division ring (green dashed lines) parallel to the microscope coverslip. (b) Representative image of a $B$. subtilis cell expressing FtsZ-GFP as a sole copy of FtsZ from the native locus (strain $\mathrm{SH} 130^{12}$ ). The division ring appears as a circle. Scale bar: $500 \mathrm{~nm}$. (c) Kymograph of FtsZ-GFP treadmilling dynamics from the cell in (b) around the cell circumference, during perturbation with the FtsZ-specific inhibitor PC190723 (cyan line). Two full revolutions around the cell $\left(0-720^{\circ}\right)$ are plotted side-by-side to resolve filament trajectories that cross $0^{\circ} / 360^{\circ}$, separated by a yellow dotted line. Diagonal lines pre-treatment show directional motion around the full circumference of the cell, while horizontal lines post-treatment show static clusters. Raw data from Whitley, Jukes et al. (2021) $)^{12}$.

We believe that a major potential application of VerCINI is the imaging of cell pole-localizing proteins. Vertical orientation increases the spatial resolution of cell poles, and high signal-to-noise imaging with TIRF illumination becomes uniquely possible as the cell pole is placed in contact with the microscope coverslip. To illustrate this we provide brief proof-of-concept demonstration of VerCINI to cell pole imaging by investigating the dynamics of the $B$. subtilis chemoreceptor protein TlpA (Figure 2). TIpA is a chemoreceptor protein in $B$. subtilis that forms large clusters localized to both the base of division septa and cell poles (Figure 2a-b), likely due to a binding preference for regions of high membrane curvature ${ }^{18}$. With conventional imaging, the distribution and dynamics of these clusters are obscured by the signal from overlapping clusters (Figure $2 b$, top). In contrast, orienting cells vertically with VerCINI and illuminating via TIRF allows for high resolution, high SNR imaging of these clusters at cell poles (Figure $2 \mathrm{~b}-\mathrm{d}$ ). We observed that TlpA forms multiple large, essentially immobile clusters (generalized diffusion coefficient $\left\langle K_{\alpha}\right\rangle=5 \cdot 10^{-7} \pm 6 \cdot 10^{-7} \mu \mathrm{m}^{2} / \mathrm{s}^{\alpha}$ (mean $\pm \mathrm{SD}$ ) for the data shown in Figure 2) of varying size, consistent with large chemoreceptor arrays observed in other organisms by cryo-electron tomography ${ }^{19}$ (Figure $2 \mathrm{~b}-\mathrm{d}$, bottom).

\section{Nanofabrication of micropillars}

The first step in VerCINI is fabrication of a silicon micropillar mould used to form the microhole cell traps. We initially designed square microholes, as we hypothesized that a deformable material like agarose should trap cells more efficiently than circular ones due to a small number of cell-microhole contact points, leading to a larger fit tolerance. Since we found that square microholes trapped cells efficiently, we have not yet investigated trapping efficiency of different trap shapes. The following protocol describes how to make a mould with arrays of square micropillars. The micropillars are made in a class 10000 cleanroom using nanofabrication techniques, specifically e-beam lithography and deep reactive ion etching (DRIE). Briefly, a silicon wafer is first spin-coated with a negative e-beam resist. An e-beam system then writes an array-of-squares pattern in the resist by exposing specified regions to an electron beam. The non-exposed resist is dissolved away using developer, leaving an array of resist squares. The resist then protects this array-of-squares pattern from being etched by vertical impinging reactive ions. All regions of the wafer with bare silicon (i.e. without resist) are etched vertically 
a

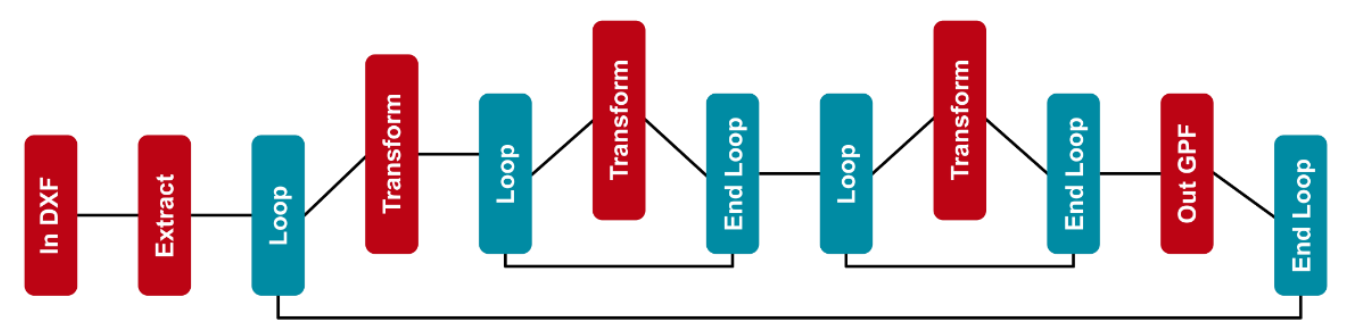

b SUBSTRATE
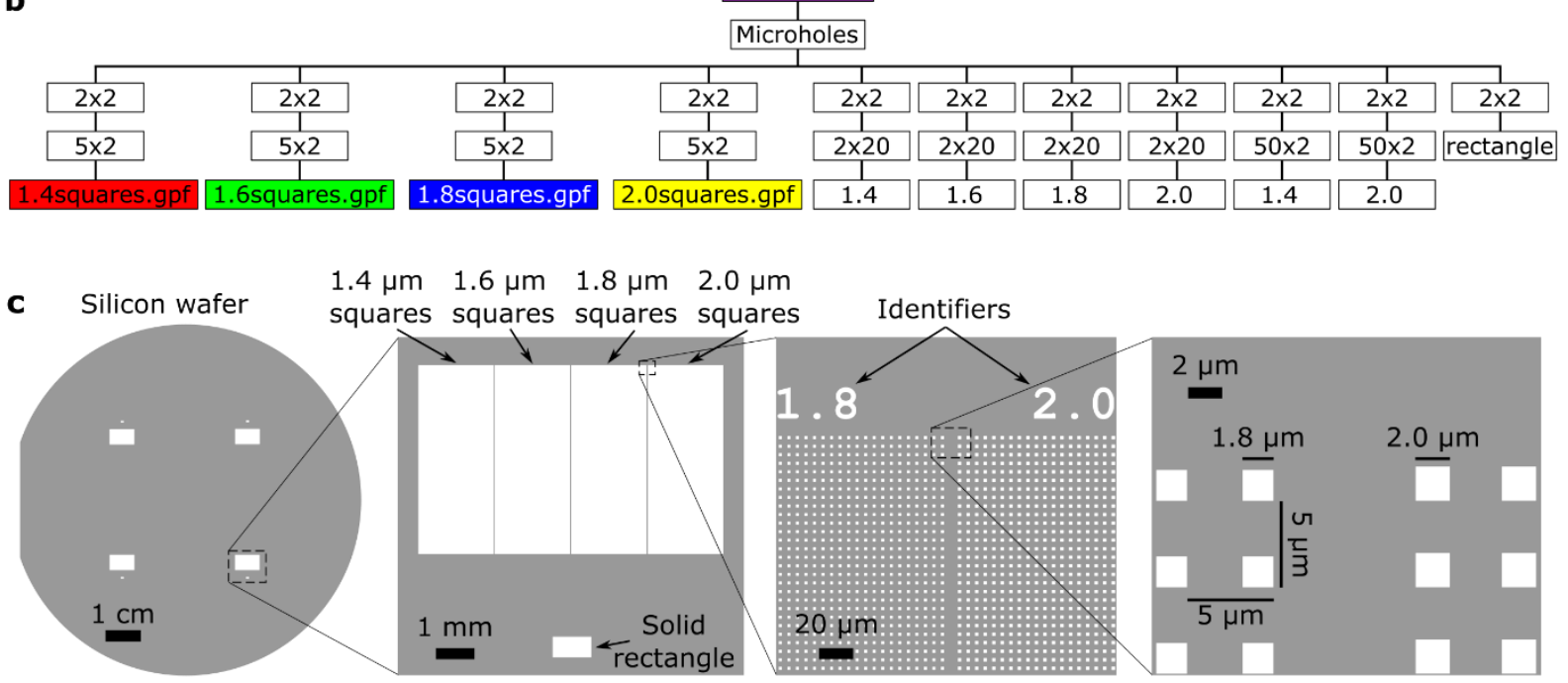

Figure 4: Design of micropillar wafer. (a) Algorithm of LayoutBEAMER used to produce arrays of squares the size of the e-beam machine's main field. (b) Algorithm of Cjob used to produce full arrays of squares of four different sizes in each quadrant of a wafer, along with identifiers and solid rectangle for measuring height after etching. (c) Output of Cjob code and overall design of silicon wafer showing arrays of squares of four different sizes in each quadrant of the wafer, along with identifiers and solid rectangle.

downward using DRIE, leaving vertical, square silicon pillars with resist on top. The resist is then removed with $\mathrm{O}_{2}$ plasma to leave bare silicon pillars. Finally, to prevent substances like PDMS from damaging the pillars when peeled off, the pillars are coated by adding a thin layer of a passivating silane compound using vapour deposition. Below, we describe the step-by-step procedure to manufacture the micropillar wafers from designing the patterns in software to silanization of the wafer.

\section{Pattern design}

Prior to any cleanroom work, the array-of-squares pattern must first be designed and exported to the e-beam system as a write job. Since each $\varnothing 100 \mathrm{~mm}$ silicon wafer can easily be split into four quarters, we recommend making four identical patterns-one in each quadrant (Figure 4c)-to maximise the utility of the wafer. Furthermore, when using VerCINI for the first time, we recommend making several columns of differently-sized pillars (Figure 4c) to later find the optimal size for your particular bacterial strain and growth conditions. For B. subtilis we have found that pillars of width 1.0-1.3 $\mu \mathrm{m}$ work best. The example pattern in Figure $4 \mathrm{c}$ is an array with four columns, each of different designed pillar width $(1.4-2.0 \mu \mathrm{m})$. Although space is available on the wafer to accommodate larger arrays, we use an overall size of $\sim 1 \times 0.5 \mathrm{~cm}^{2}$ because the agarose pad that will eventually contain this pattern will need to be cut in size prior to imaging to ensure sufficient oxygen delivery to trapped cells (see Figure 6avi). In addition to the array-of-squares pattern, identifiers labelling the pillar widths are also included immediately above the squares so their widths can be readily identified under a microscope (Figure 4c). Additionally, a solid rectangle of $1000 \times 500 \mu \mathrm{m}^{2}$ is also included to the pattern to facilitate measurement of the heights of pillars after etching. 
One factor when designing this pattern is e-beam write time. Since it takes significantly more time for the e-beam system to move the sample stage than to deflect the beam, moving the stage to write each individual square shape will require a prohibitively long amount of time. However, the area over which the e-beam can write at a single stage position (the main field; $1 \times 10^{6} \mu \mathrm{m}^{2}$ for our Raith EBPG-5000+) is much larger than each individual square that will be written $\left(\sim 1 \mu \mathrm{m}^{2}\right)$. So, it is best to first design an array of squares that is roughly the same size as the e-beam's main field, and then later replicate this array as many times as needed to obtain a larger array pattern. This way, thousands of squares are written at each stage position, dramatically reducing the e-beam write time.

The initial square pattern is made in a software such as AutoCAD (Autodesk). This square is then replicated into an array of squares the size of the e-beam main field using LayoutBEAMER (GenISys $\mathrm{GmbH}$; algorithm shown in Figure 4a). For the Raith EBPG-5000+ e-beam system the main field is 1048 $\times 1048 \mu \mathrm{m}^{2}$. LayoutBEAMER will also convert the CAD file to a pattern of dots for the e-beam. Note that we do not use proximity effect correction for this procedure, as the features are large enough that we have found this unnecessary. The output array pattern is then replicated to form the larger array, along with identifiers and solid rectangle, in Cjob (Vistec Lithography; algorithm shown in Figure 4b). The output of $\mathrm{Cjob}$ is an exposure script for the e-beam system (Figure 4c).

Importantly, we have found that the etching process shrinks the squares substantially (Figure 5a-b) from the defined width, so when choosing the sizes of the pillar widths, these must be increased to compensate for this effect. For example, when $1.3 \mu \mathrm{m}$ square pillars are desired, users should design squares of $\sim 2.0 \mu \mathrm{m}$. We have found that the decrease in size is reproducible for the pillar design described in this protocol ( $\mathrm{N}=6$ wafers). However, the amount that pillars shrink during etching shows a non-linear dependence on the size of the gaps between them, so for closely spaced pillars this must be considered (Figure 5c).

Protocol for Micropillar pattern design:

1. Make a $1 \times 1 \mu \mathrm{m}^{2}$ square in AutoCAD. Save it as a dxf file.

2. In LayoutBEAMER, make an array of squares the size of the e-beam main field. One possible algorithm to achieve this is shown in Figure 4a.

a. Place "Import". In the pop-up GUI choose the dxf file containing the square. This block will then change to "In DXF".

b. Place "Extract".

c. Use one loop to scale the $1 \times 1 \mu \mathrm{m}^{2}$ square to the desired sizes. Each will be output as its own gpf file.

d. Within this loop, make two more loops in series to make an array of squares. Note that with LayoutBEAMER loops you can choose to either keep the result of the final iteration or merge the results of all loops. We use the latter for speed, and so these two loops are in series rather than having one nested within the other.

e. Place "Export". This changes to "Out GPF" after choosing to save it as a gpf file.

f. Press the Play symbol on the last End Loop to execute the code.

g. Inspect the output in LayoutBEAMER. Verify that the pattern fits within the main field.

3. In Cjob: Load your array from LayoutBEAMER and replicate it several times to make the full arrays. Also add in identifiers and a solid rectangle to measure heights. A code structure to achieve this is shown in Figure 4b.

a. Place "Substrate".

b. Place "Exposure". Use 100 kV. 
c. Place "Layout". Repeat the pattern twice in the $X$ direction and twice in the $Y$ direction to make identical copies in each quarter of the wafer.

d. Place another "Layout" beneath this one. Repeat the pattern twice in the X direction and five times in the $Y$ direction to expand the size of each array tenfold.

e. Place "Pattern" beneath this. Load one of your gpf files and choose the appropriate dose and beam size. The dose is specific to each resist; for AR-N-7700 this is 117 $\mu \mathrm{C} / \mathrm{cm}^{2}$. We use a beam step size of $25 \mathrm{~nm}$ and beam size of $56 \mathrm{~nm}$.

f. Repeat this for multiple square sizes.

g. Add identifiers around the entire array so that the sizes can be identified later under a microscope. To do this, also place two "Layouts", and beneath these place "Identifier". Since precision is not important in this case, choose the largest beam size and low resolution (we use a beam step size of $50 \mathrm{~nm}$ and beam size of $95 \mathrm{~nm}$ ). Since the e-beam system takes time to change the beam size, it is best to write the identifiers last rather than after each square size is finished.

h. Add a solid square above each array so that the heights can be measured with a profilometer after etching. To do this, place another $2 \times 2$ "Layout" and beneath this place "Shape". Choose a shape of $1000 \times 500 \mu \mathrm{m}^{2}$, and leave the beam step size and beam size the same as for identifiers.

i. Save the file as a .cjob file.

j. Export the file as a .job file. Use the button Exposure Time to estimate how long the job will take to write. For us this has typically been $\sim 50 \mathrm{~min}$.

\section{Resist spin-coating}

Prior to spin-coating, the wafer (4 inch diameter, $\sim 500 \mu \mathrm{m}$ thickness, one side polished, type/orientation $\mathrm{NP}<100>, \mathrm{PB}<100>$, resistivity $1-10 \Omega \cdot \mathrm{cm}$; International Wafer Service) can optionally be cleaned with fuming nitric acid to remove dust and impurities. This is recommended to prevent non-uniform deposition of resist on the wafer during spin-coating and hence ensure that the patterns avoid defects arising from this. However, we have found that this step is typically unnecessary if fresh silicon wafers are used straight from packaging.

The wafer must be coated with negative resist in which the e-beam can write patterns. The wafer is primed with 1,1,1,3,3,3-hexamethyldisilazane (HMDS; VWR, cat. no. 51152885) to increase adhesion of the resist, then coated with the resist itself.

The following protocol is specific for the AR-N-7700.18 resist (Allresist $\mathrm{GmbH}$ ) that we used. If using another type, use the appropriate bake temperature and spin settings for that resist.

Protocol for resist spin-coating:

1. Set one hot plate to $200^{\circ} \mathrm{C}$ and another to $85^{\circ} \mathrm{C}$.

2. Set wafer on spin chuck and pull vacuum.

3. Blow off dust from wafer with compressed air.

4. Spread $\sim 5 \mathrm{~mL}$ HMDS on the wafer, trying to avoid bubbles.

5. Spin at $1000 \mathrm{rpm}$ for $1 \mathrm{~min}$.

6. Bake wafer for $2 \mathrm{~min}$ at $200^{\circ} \mathrm{C}$.

7. After wafer cools down, set back on spin chuck and pull vacuum.

8. Using a syringe with $0.22 \mu \mathrm{m}$ filter, carefully spread $\sim 5 \mathrm{~mL}$ filtered resist on wafer, avoiding bubbles as much as possible. Push any bubbles away from the regions of the wafer where the e-beam will be writing patterns.

9. Spin at $500 \mathrm{rpm}$ for $1 \mathrm{~min}$. 
10. Bake wafer for $2 \mathrm{~min}$ at $85^{\circ} \mathrm{C}$.

\section{Micropillar pattern writing}

Patterns are written using electron-beam lithography. Exposure to the e-beam alters the solubility of the resist by inducing polymer crosslinking. This reaction is later completed with a post-exposure bake of the wafer. Failure to perform a post-exposure bake can result in all features dissolving during the subsequent development step.

The following protocol is specific for the EBPG-5000+ e-beam system (Raith $\mathrm{GmbH}$ ), although it can easily be adapted for others.

Protocol for micropillar pattern writing:

1. Fit the resist-coated wafer onto an appropriate holder.

2. At the e-beam, press "Set Lock Vent".

3. When ready, slide in the wafer with holder and seal the chamber.

4. Press "Set Lock Vacuum".

5. When the system is ready, start the writing job.

6. When finished, press "Set Lock Vent" again.

7. Remove the wafer and bake it for $2 \mathrm{~min}$ at $105^{\circ} \mathrm{C}$.

\section{Development}

The goal of this step is to dissolve and remove all unexposed resist (i.e. all resist that was not exposed to the e-beam). Before doing this the wafer must be baked (see protocol for resist described above) or the pattern itself may be removed by the developer.

In this procedure, the wafer is rinsed in three steps: concentrated developer, diluted developer, and water. If left too long in developer, even the exposed resist will dissolve. So, the timing is important to ensure that the designed pattern, and only the designed pattern, remains on the wafer.

Protocol for development:

1. Set up three wide beakers for submerging and rinsing the wafer. In the first, pour enough Microposit MF-321 developer (micro resist technology $\mathrm{GmbH}$ ) to submerge the wafer. In the second, pour a 1:9 ratio of MF-321:water (e.g. $50 \mathrm{~mL}$ MF-321 and $450 \mathrm{~mL}$ water). In the third, fill with a large amount of water to fully dilute any remaining resist on the wafer surface.

2. Dip the wafer into the beakers sequentially with the following timing, gently swirling the solution:
a. Beaker 1 (MF-321): $90 \mathrm{~s}$
b. Beaker 2 (MF-321:water): $30 \mathrm{~s}$
c. Beaker 3 (water): $>30 \mathrm{~s}$

3. Remove the wafer from the water beaker, rinse off with more water, and spin-dry.

4. Use a light microscope with 60-100 x magnification to inspect the patterns and verify that the unexposed resist has been removed. If there is still a significant amount remaining, develop the wafer another $\sim 30 \mathrm{~s}$, rinse with water, dry, and inspect again.

\section{Etching and inspection}

The goal of etching is to produce pillars of a specific height. The optimal height to use is mainly determined by the average length of cells that users want to eventually image in microholes. For example, if a researcher wants to use VerCINI with cells that are on average $4 \mu \mathrm{m}$ long, it would be 
a
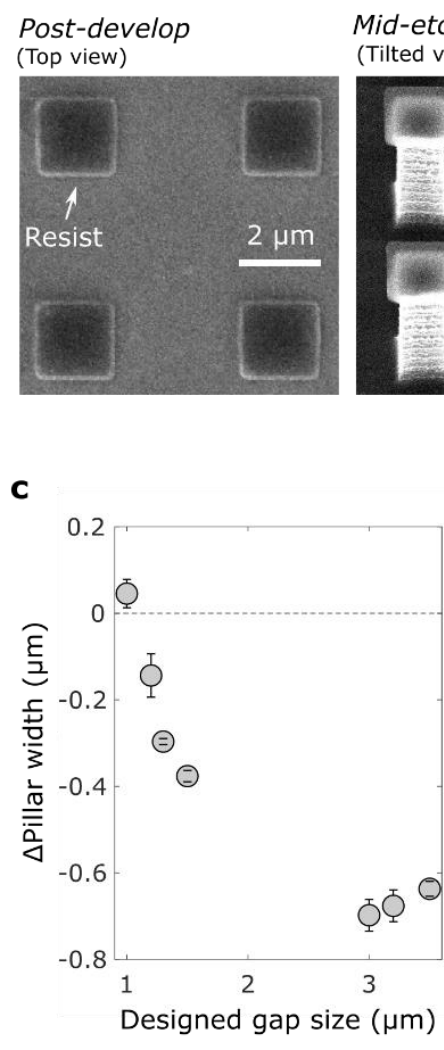

Mid-etch (Tilted view)

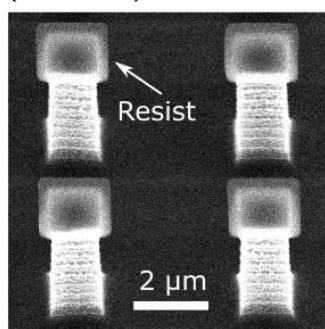

d

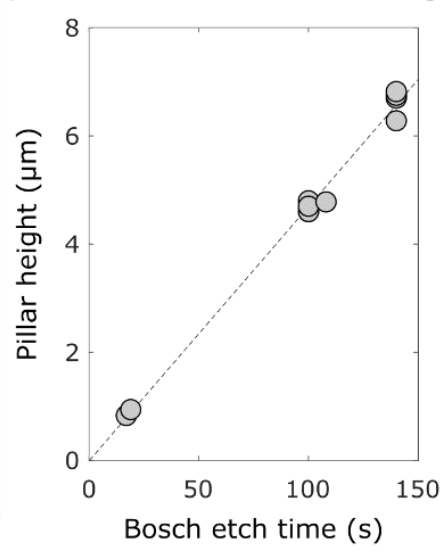

b

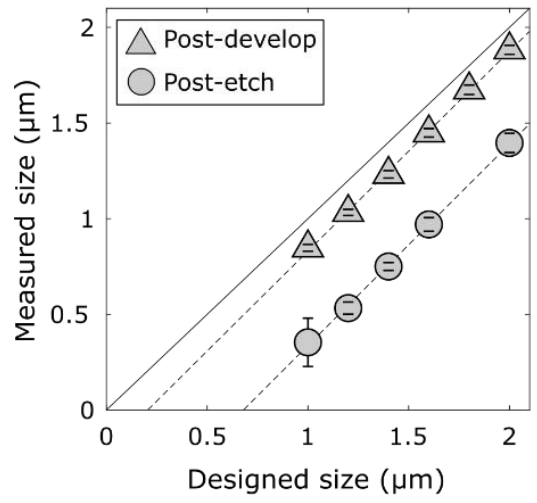

Final product (Tilted view)

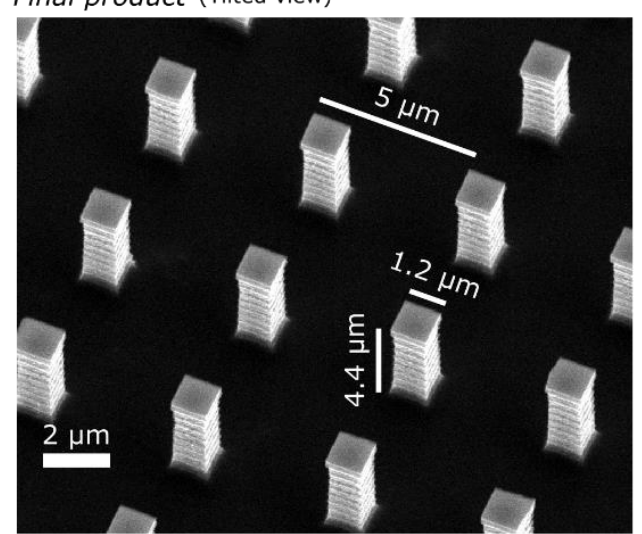

Figure 5: Development and etching of micropillar wafer. (a) SEM image of silicon wafer with squares with designed edge lengths of $2.0 \mu \mathrm{m}$ during development and etching. Left panel: Patterned squares of e-beam resist remaining after development, seen from top down. Middle panel: Patterned squares after several cycles of Bosch etch, but before removing resist with oxygen plasma, seen from a tilted angle. Etching produces significant undercutting. Right panel: Patterned squares after etching and oxygen plasma to remove resist, seen from top down. Scale bars: $2 \mu \mathrm{m}$. (b) Comparison of designed square sizes to measured square sizes after development (triangles) and after $100 \mathrm{~s}$ of Bosch etch (circles). Solid line shows hypothetical 1:1 correlation. Dotted lines show linear fits to post-develop and post-etch data. Error bars are SD. (c) Change in widths of pillars after $140 \mathrm{~s}$ Bosch etch compared to designed size of gaps between pillars. Error bars are SD. (d) Effect of etch duration on final heights of micropillars. Each circle represents a separate wafer or wafer quarter. Wafers had different square sizes, but all had spacing of $5 \mu \mathrm{m}$. Dotted line: linear fit to data. (e) SEM image of a final silicon micropillar wafer with measured widths $\sim 1.2 \mu \mathrm{m}$ and spacing $5 \mu \mathrm{m}$ (and hence gap of $3.8 \mu \mathrm{m}$ between pillars). Heights were measured to be $4.4 \mu \mathrm{m}$ from profilometer.

wise to make the pillars at least $4 \mu \mathrm{m}$ tall. Pillars can also be made taller to accommodate longer cells or multiple short cells as a column, but there is a limit to how tall the pillars can be. If the aspect ratio of pillars becomes too high they can become fragile and more susceptible to breaking when agarose or PDMS are peeled off of them. We have successfully used pillars with aspect ratio up to 10 $(1 \mu \mathrm{m}$ square pillars $10 \mu \mathrm{m}$ tall), and it is likely that much higher aspect ratios will not be feasible.

This protocol uses an inductive coupled plasma (ICP) reactive ion etcher (Adixen AMS 100 I-Speeder). Etching is done with a Bosch process, which uses alternating infusions of gases $\mathrm{SF}_{6}$ and $\mathrm{C}_{4} \mathrm{~F}_{8}$ that are ionized by RF power to etch and passivate the bare silicon surface, respectively, while regions covered with resist are unaffected. A potential bias accelerates the ionized gases downward onto the wafer to produce a vertical etch. Each cycle of etching/passivating increases the heights of the pillars, and so the duration of this Bosch process determines the heights of the pillars (Figure $5 \mathrm{~d}$ ). Although the goal of this procedure is to produce a strictly vertical etch, we have found that it causes the square pillars 
to laterally shrink significantly from their designed sizes (Figure $5 a-b)$ due to some degree of 'isotropic etching' (i.e. undercutting the resist). The degree to which the pillars shrink does not appear to depend on the designed square sizes (Figure $5 b$ ) so long as the gap between pillars is sufficiently large. However, the amount that pillars shrink during the Bosch etch shows a non-linear dependence on the size of the gaps between pillars (Figure $5 \mathrm{c}$ ), and for closely spaced pillars this must be taken into consideration. The duration of the Bosch etch also appears to have some effect on the final pillar widths: we have measured a decrease in widths of $560 \pm 110 \mu \mathrm{m}$ (mean \pm SD) for a $100 \mathrm{~s}$ Bosch etch ( $\mathrm{N}=4$ wafers) and $680 \pm 40 \mu \mathrm{m}$ (mean $\pm \mathrm{SD}$ ) for a $140 \mathrm{~s}$ Bosch etch ( $\mathrm{N}=2$ wafers).

Following the etch, the resist is removed by $\mathrm{O}_{2}$ plasma. After the resist is removed, the pillar heights can no longer be increased. So, if establishing a new Bosch process duration to get specific pillar heights it may be advantageous to eject the wafer before $\mathrm{O}_{2}$ plasma treatment and measure the pillar heights so that further etching can still be done if needed. If doing so, it is helpful to correct for the thickness of the resist, which adds a small extra height (for AR-N-7700.18 resist this is $\sim 180 \mathrm{~nm}$ ).

Heights are measured using a stylus profilometer (Bruker Dektak XT). This device measures the heights of features by sensing the deflection of a fine stylus as it moves over the surface. To accurately measure heights without damaging the pillars, we move the stylus over the large $\left(1000 \times 500 \mu \mathrm{m}^{2}\right)$ solid rectangle that was included in the pattern for this purpose.

Protocol for etching and inspection:

1. Clean the chamber of the etcher for 20 min prior to beginning using $\mathrm{O}_{2}$ gas set to 200 standard cubic centimeters per min (SCCM) with ICP power set to $1800 \mathrm{~W}$ and biased power set to 60 W.

2. Make a recipe for etching.

a. Set wafer temperature to $10^{\circ} \mathrm{C}$, chamber pressure to $0.04 \mathrm{mbar}$, and sample holder at $200 \mathrm{~mm}$ from the plasma source.

b. Set the etching step to $200 \mathrm{SCCM} \mathrm{SF}_{6}$ for $7 \mathrm{~s}$ with ICP power at $2000 \mathrm{~W}$ and biased power at $0 \mathrm{~W}$.

c. Set the passivation step to $80 \mathrm{SCCM} \mathrm{C} \mathrm{C}_{4}$ for $3 \mathrm{~s}$ with ICP power at $2000 \mathrm{~W}$ and CCP power in chopped low-frequency bias mode: $80 \mathrm{~W}$ for $10 \mathrm{~ms}$ and $0 \mathrm{~W}$ for $90 \mathrm{~ms}$.

d. Under the tab "step time", modify the full process time to the duration needed to achieve desired pillar heights (use Figure $5 \mathrm{~d}$ as a guide).

3. Make a recipe for removing the resist.

a. Set wafer temperature to $10^{\circ} \mathrm{C}$, chamber pressure to $0.04 \mathrm{mbar}$, and sample holder at $200 \mathrm{~mm}$ from the plasma source.

b. Set $200 \mathrm{SCCM} \mathrm{O}$ with ICP power at $2500 \mathrm{~W}$ with biased power of $50 \mathrm{~W}$.

c. Under the tab "step time", set the time to $10 \mathrm{~min}$.

4. Etch wafer:

a. Vent loadlock.

b. Place wafer on loading arm.

c. Close chamber and pump loadlock.

d. When loadlock has reached $\sim 2 \times 10^{-1}$ mbar and the valve indicator turns green, load your etching recipe and start the run.

5. Remove resist:

a. Load resist removal recipe and start run.

b. When finished, vent loadlock.

6. Remove wafer and measure the pillar heights with the profilometer. 
7. Since the etching procedure typically reduces the widths of the pillars, it is important to inspect their true sizes with SEM at the end of the process. For this we have used a Nova NanoSEM (FEI).

\section{Silanization}

Substances like PDMS tend to adhere strongly to the surfaces of silicon wafers, making it difficult or in some cases nearly impossible to remove them without destroying the delicate pillar patterns. To avoid this, it is common practice to coat the wafers with a silane compound (tridecafluoro-1,1,2,2tetrahydrooctyl trichlorosilane; abcr $\mathrm{GmbH}$ ) by vapour deposition to prevent strong adhesion. Although primarily to enable solidified PDMS to be removed, we have also found that this silane coat makes solidified agarose peel off more easily as well. Note: since this silane compound reacts with water, it must be kept strictly anhydrous using an inert gas like argon.

Protocol for silanization:

1. Prepare a glass desiccator with inlet tubing for argon gas flow. The argon gas will evacuate air from the chamber to remove moisture.

2. Place the wafer into the argon-filled desiccator.

3. Place a small $600 \mu \mathrm{L}$ tube in the desiccator and leave it open.

4. Place bottle of silane compound in desiccator.

5. Open the bottle and pipet $10 \mu \mathrm{L}$ silane compound into the $600 \mu \mathrm{L}$ tube. Leave the tube open.

6. Close the silane bottle and seal with parafilm.

7. Turn off argon gas and remove the tubing.

8. Pull vacuum in desiccator (down to $\sim 10 \mathrm{mbar}$ ).

9. Close the desiccator valve and leave overnight.

\section{Wafer dicing}

Optionally, the wafer can be cleaved into four separate quadrants using a diamond scribe (RS Pro, Stock No. 394-217). Since the wafers are made of a lattice crystal of silicon, they will break along a well-defined plane. This is done by scratching a notch with the diamond scribe at the edge of the line the wafer will be split along, and then applying force to break the wafer. If done properly the crack should immediately propagate across the wafer and cleanly break it.

\section{Sample preparation and imaging}

In this section, we describe how to make agarose microhole pads from the silicon moulds, load cells into them, and image. To image cells during vegetative growth, VerCINI pads are made using agarose dissolved in growth media. The porous nature of the agarose gel allows nutrients to diffuse through to cells allowing continued growth during the imaging process, while a high agarose concentration makes the gel stiff, providing structural stability to the microholes.

One critical factor for VerCINI is the efficiency with which cells are loaded into holes. A higher loading efficiency corresponds to more trapped cells in a single FoV, and therefore greater data acquisition. We have found that centrifuging concentrated cell culture into the holes using a flat bottomed centrifuge rotor gives high loading efficiency (Figure $6 c-d$ ).

It is also important to consider are the morphology and phenotype of the cells that are being used. Cells that are filamentous or strongly curved are not likely to fit in the agarose holes. However, even cells with a rod-shaped morphology can have characteristics that pose challenges to VerCINI. For 
a

i. Apply agarose to wafer
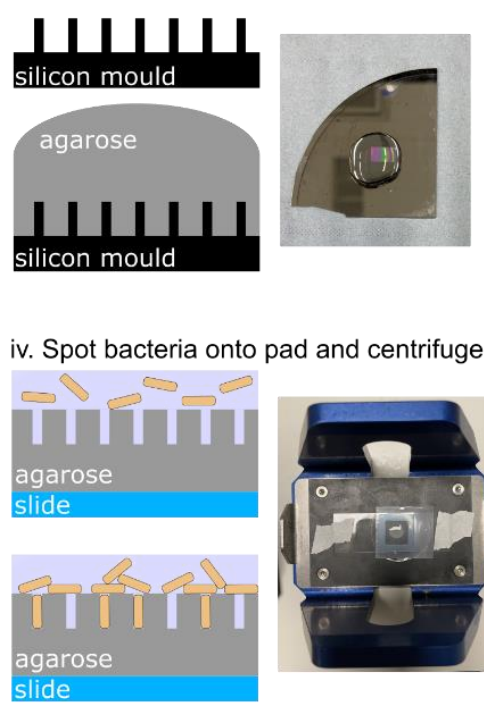

b

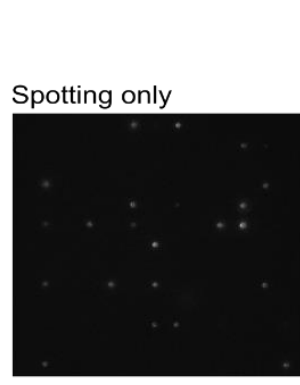

Spotting, washing ii. Apply slide onto agarose

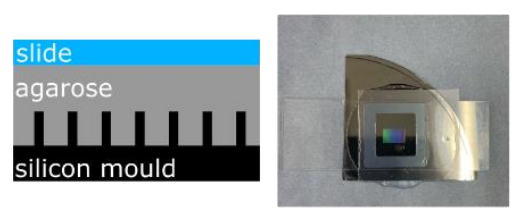

v. Wash

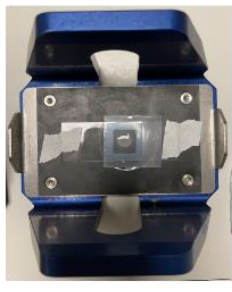
centrifugation,

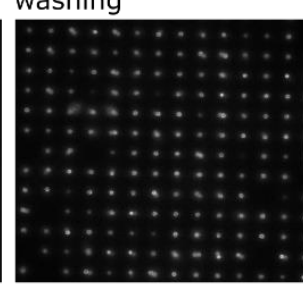

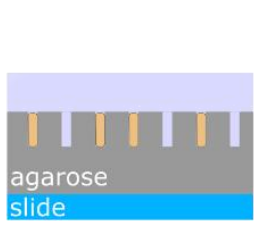

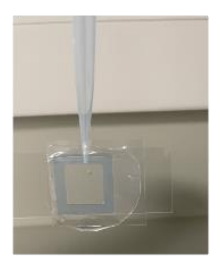

iii. Remove wafer

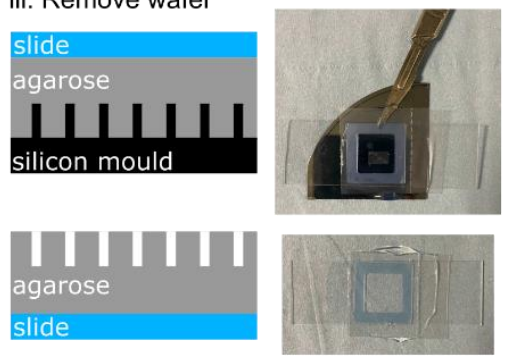

vi. Remove excess agarose and apply cover glass

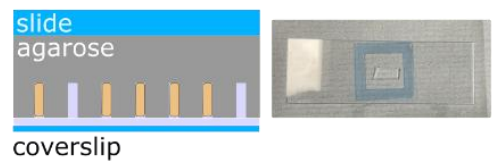

Figure 6: Sample preparation for VerCINI. (a) Sample preparation workflow. (i) Molten agarose is applied to the silicon micropillar wafer. (ii) The cover slide is applied onto the agarose with Gene Frame down. (iii) The micropillar wafer is removed from the agarose slide. (iv) Concentrated bacteria are pipetted onto the imprinted agarose, and the slide is centrifuged to increase loading efficiency. (v) Excess horizontal cells are washed off the pad. (vi) Excess agarose is cut away from the pad, leaving only the microhole imprinted area and the cover glass is applied. (b) HILO VerCINI of $B$. subtilis cells expressing FtsZ-GFP (strain $\mathrm{SH} 130^{12}$ ) loaded into microholes with or without centrifugation. Left: cells loaded only by spotting liquid culture onto the VerCINI pad and applying the coverslip. Right: cells loaded by spotting concentrated liquid culture, centrifuging, and washing off unloaded cells. (c) Comparison of loading efficiency between the two loading methods shown in (b). (d) Brightfield images of $\mathrm{SH} 130$, before and after the washing step.

example, we have found that cell chaining-a phenotype characterized by long chains of cells that do not separate after completing division-can limit the use of VerCINI in the B. subtilis PY79 background. Like filamentous cells, these long chains do not fit in VerCINI microholes, preventing data acquisition. However, we have found that deleting the hag gene that encodes flagellin reduces chaining compared with wild-type. Alternatively, deletion of the transcriptional regulation gene $s / r R$ also reduces chaining in this background, as previously shown ${ }^{20}$.

Another important consideration that we have observed is that cells are sometimes poorly trapped in the holes, causing them to wobble. Such imperfectly-trapped cells can be identified and removed from further analysis by recording a short bright-field video after fluorescence acquisition. We initially hypothesized that flagellar motility might cause this poor trapping. However, at least in B. subtilis, this does not appear to be the case. Poorly trapped $B$. subtilis cells are relatively rare for correctly sized micropillars. As deleting the hag gene made little difference to the amount of poorly trapped cells, 
eliminating flagellar motility does not appear to be necessary for VerCINI microscopy. In the less chainforming B. subtilis strain BS168 we frequently perform experiments in motility-proficient cells without issue.

Since microholes are imprinted on the top of the agarose pad, the cells are adjacent to the coverslip surface when the coverslip is applied. Cells can then be imaged using any high resolution microscope technique suitable for studying bacterial spatial organization. Illumination techniques such as HILO (for cell sidewall) and TIRF (for cell poles) are preferable to maximize image signal to noise ratio (SNR).

\section{VerCINI agarose pad preparation}

To prepare an agarose VerCINI pad, we use $6 \%$ agarose dissolved in media. The molten agarose is spotted onto a micropillar mould, then a microscope slide is firmly pressed down to produce a flat agarose pad (Figure 6a). After the agarose solidifies, the wafer is then removed, leaving the microhole pattern imprinted on the agarose pad. The wafer should only be removed immediately before cell loading to prevent dehydration of the pad.

Protocol for VerCINI agarose pad preparation:

1. Prepare a $90^{\circ} \mathrm{C}$ water bath.

2. Apply a Gene Frame ( $65 \mu \mathrm{L}, 1.5 \times 1.6 \mathrm{~cm}^{2}$; Thermo Scientific) to a microscope slide (VWR Super Premium). Leave the plastic cover adhered to the Gene Frame that has a square hole in the middle.

3. Prepare $10 \mathrm{~mL}$ of $6 \%$ agarose in growth media (with any inducers required) and microwave until dissolved. Short bursts in the microwave with swirling in between allow bubbles to settle.

4. After agarose is fully dissolved, place the molten agarose in the $90^{\circ} \mathrm{C}$ water bath for $5-10 \mathrm{~min}$. Since the $6 \%$ molten agarose is very viscous, this will allow time for bubbles to migrate to the surface while not allowing the agarose to solidify.

5. Optionally, place the micropillar wafer and microscope slide on a hotplate set to $50^{\circ} \mathrm{C}$. The higher temperature will give more time for the agarose to solidify while pressing down to make the pads, making it easier for users to make the pads sufficiently thin.

6. Using wide-bore pipette tips (or cutting the ends off regular ones), apply $800 \mu \mathrm{L}$ agarose to the centre of the pillars (Figure 6ai).

7. Gently but firmly press the microscope slide on top of the agarose (Gene Frame down), aligning the micropillars with the centre of the Gene Frame (Figure 6aii).

8. Leave the micropillar wafer in place on the agarose at imaging temperature until you are ready to load with cells.

\section{Cell cultivation and VerCINI pad loading}

To minimize possible perturbations to cell physiology we recommend that cell culture and microscopy be performed at identical temperature, and in the same media. VerCINI sample preparation steps such as agarose pad spotting and centrifugation should also be preferably be performed at the cell culture temperature. If this is not possible, the time for which cells are brought to room temperature for these steps should be kept to a minimum. Cells should preferably be prepared in a low autofluorescence medium to maximize image quality.

Although cells can be loaded into holes by simply spotting liquid culture on the agarose pad and applying a coverslip, we have found that this typically leads to low loading efficiency (Figure $6 c-d$ ). We 
found that concentrating and centrifuging cells into the holes using a flat bottomed centrifuge rotor improves loading efficiency (Figure 6c-d).

Washing the excess cells from the pad is a critical step in this process. If the washing step is not thorough enough, the pad will be covered in horizontal cells covering up the vertically immobilized cells (Figure 6b). If the pad is washed too aggressively, the vertically immobilized cells will be flushed out of the microholes. The washing step is also important to maintain hydration of the pad after centrifugation. Slides are transported to the microscope in an empty pipette tip box which has been pre warmed at imaging temperature to reduce temperature instability.

Protocol for cell cultivation and VerCINI pad loading:

1. Using a scalpel, remove agarose slide from the micropillar wafer (Figure 6aiii). To do this, slide the scalpel between the agarose pad and the wafer, then use the scalpel to lever the agarose pad and slide away from the wafer. Take care not to disturb the holes or to touch the nanofabricated pillars.

2. When cells are between $\mathrm{OD}_{600}$ of $0.3-0.5$, centrifuge $500 \mu \mathrm{L}$ of cell culture at $17,000 \mathrm{rcf}$ for 1 min. Concentration and volume of cells added can be adjusted as required.

3. Remove supernatant and resuspend in $8 \mu \mathrm{L}$ pre-warmed media.

4. Apply the concentrated cells to the centre of the imprinted area of the agarose pad (Figure 6aiv). To prevent the culture from smearing and drying out, the concentrated culture can be placed inside a $9 \mathrm{~mm}$ silicone gasket (Sigma-Aldrich, Cat No. GBL103240) placed on top of the holes.

5. Tape the slide to a flat-bottomed (for 96-well plates) centrifuge rotor with appropriate balance.

6. Centrifuge at 3,220 rcf for 4 minutes (Figure 6aiv).

7. To wash off excess cells from the top of the pad, hold the pad near-vertically over a waste container (Figure 6av). $1 \mathrm{~mL}$ at a time, slowly $(1 \mathrm{~mL} / 5 \mathrm{~s})$ pipette fresh, pre-warmed media onto the top of the agarose pad, allowing the media to flow over the imprinted area and drop into the waste container.

8. Repeat this step at least 5 times until the majority of excess cells are removed (it is impossible to remove all of the cells so some excess will still be visible by eye). This step is the most inconsistent, so can be adjusted through trial and error.

9. Allow the pad to air dry until no excess liquid remains ( $\sim 2$ minutes).

10. Using a scalpel, cut around the imprinted region, removing all other agarose. This is to ensure sufficient oxygen supply to the cells once the cover slip is applied (Figure 6avi).

11. Peel off the remaining plastic from the Gene Frame and apply the coverslip (VWR $22 \times 22$ $\mathrm{mm}^{2}$ Thickness no. 1.5). Ensure the coverslip is fully adhered to the Gene Frame by pressing down around all the edges.

12. Transport slides to microscope in a pre-warmed empty pipette tip box to reduce temperature fluctuations in the sample.

\section{VerCINI imaging}

The specific imaging protocol should be designed depending on the experiment and imaging setup. Since the cells are adjacent to the coverslip, high SNR illumination methods such as TIRF or HILO can be used, depending on application. TIRF can be used in some specific scenarios where the protein to be observed localises near the cell poles (e.g. Figure $2 b$, bottom). Otherwise, HILO can be used, which provides illumination of the whole cell but not the agarose above the cell, increasing the signal to noise ratio (SNR) beyond that of widefield illumination (e.g. Figures $1 \mathrm{~b}$, bottom and $3 \mathrm{~b}$ ). We employ a ringTIRF or ring-HILO system, where galvanometer-driven mirrors rotate the illumination beam at high speed $(200 \mathrm{~Hz})$ to produce uniform illumination across the sample ${ }^{21}$. 
In addition to the fluorescence video of protein dynamics, we recommend recording a short ( $1 \mathrm{~s})$ brightfield video following fluorescence acquisition so that improperly-trapped cells ('wobblers') can be easily identified and removed during analysis. Depending on the protein of interest, it may also be useful to record a fluorescence Z-stack after the fluorescence dynamics video to ensure correct Z-plane has been chosen and exclude cells with bright structures immediately above or below imaging plane. This is specifically recommended for cell division proteins.

Protocol for VerCINI imaging:

1. Apply the slide to the microscope, preferably with a low autofluorescence immersion oil such as Olympus Type-F.

2. Using brightfield illumination, identify an area of the VerCINI slide with a high loading efficiency and a low number of horizontal cells on top of the pad.

3. Using fluorescence microscopy, take snapshots to scan the Z-plane to find the structure you wish to image. Keep the number of snapshots to a minimum to avoid photobleaching. It is often useful to take a Z-stack image of the entire cell to ensure the correct structure and plane are imaged.

4. Set focus lock to the optimal Z-plane.

5. Record a short brightfield time lapse as a control. This allows later identification of wobbling cells which can affect apparent protein dynamics. We recommend recording $\sim 20$ frames over $\sim 1 \mathrm{~s}$.

6. Record fluorescence with desired settings.

\section{$\mu$ VerCINI}

In many experiments, researchers want to perform solution exchange during imaging, either to change from one medium to another, to add perturbatives, to fix cells, or to label them with exogenous dyes. However, with the original VerCINI method this is not possible since the slide is closed and the cells are underneath a fairly dense block of agarose. We have therefore designed an adapted version of the method to be compatible with solution exchange, called microfluidic VerCINI ( $\mu$ VerCINI). In this method, the microholes are open-topped, and the cells are exposed to a fluid environment that is controlled by users through a fluidic system. Below, we describe the step-by-step procedure to assemble the $\mu$ VerCINI device, load cells, and image them during solution exchange.

\section{$\mu$ VerCINI coverslip fabrication}

The first step in this technique is to form the open-topped microholes on a microscope coverslip (Figure 7a). One limitation to this orientation is that there will be material between the coverslip surface and the cells that must be imaged through. Since high-NA microscope objectives have a short working distance and cannot image very deeply, this layer must be as thin as possible. Making such a thin layer with agarose is challenging, and imaging through a thick layer of agarose would lead to substantial loss in image quality due to scattering. Instead, we found that it is beneficial to use polydimethylsiloxane (PDMS), which is commonly used for microfluidic applications. Most of the equipment we use for working with PDMS was purchased as a kit (PDMS replication station; BlackHole Lab). Since PDMS can adhere tightly to silicon, peeling PDMS-covered coverslips off of unsilanized silicon nanostructures can easily damage the structures. It is therefore essential that the silicon wafers have been silanized prior to using PDMS on them.

In this protocol, open-topped PDMS microholes are formed on a microscope coverslip. PDMS elastomer base and curing agent are mixed together and degassed to remove bubbles. The mixture is 
a $\mu$ VerCINI coverslip assembly

i. Pour PDMS

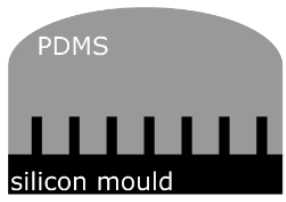

iii. Bake, peel off

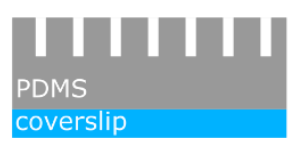

C

\section{Cell loading}
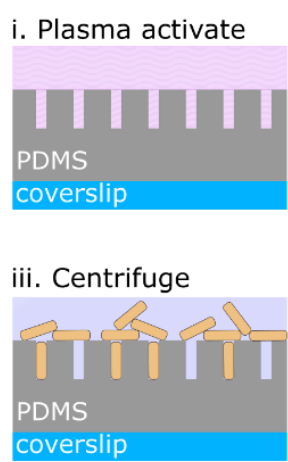

Final product ii. Flatten with coverslip
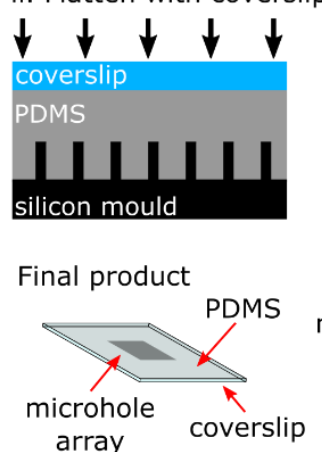

d

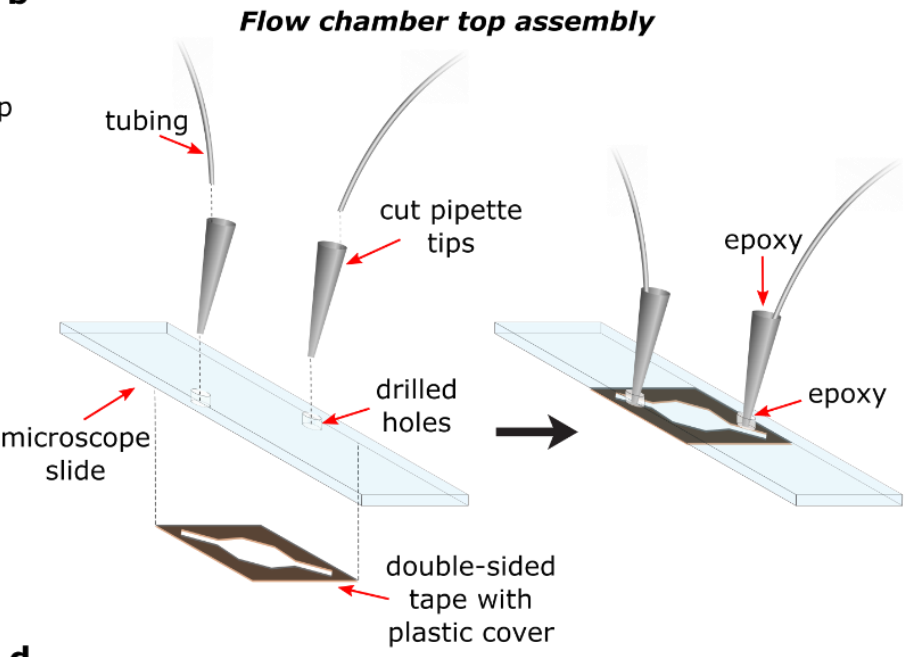

Flow chamber full assembly

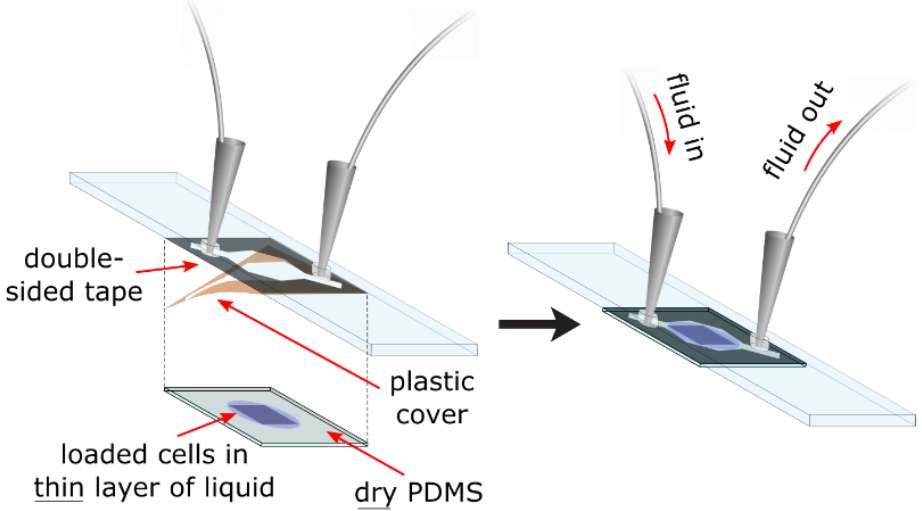

Figure 7: Device assembly and cell loading for $\mu$ VerCINI. (a) Assembly of $\mu$ VerCINI coverslip. (i) Degassed PDMS in a 10:1 elastomer base : curing agent ratio is poured onto silicon micropillars. (ii) A coverslip is pressed firmly down on top of the PDMS to form as thin a layer as possible. (iii) The PDMS is baked in an oven and peeled off to produce a coverslip with open-topped PDMS microholes. The final product can be stored for months to years. (b) Assembly of top of flow chamber. A microscope slide has holes drilled into it. Cut pipette tips are inserted into these holes and epoxied in place, and tubing is inserted into the cut pipette tips and epoxied in place. A piece of double-sided tape with plastic cover still attached has a groove cut into it and is adhered to the microscope slide. The final product can be stored for months to years. (c) Loading cells into open-topped microholes. (i) PDMS is rendered hydrophilic through treatment with air or oxygen plasma. (ii) Cell culture is concentrated and added on top of the microholes. (iii) The $\mu$ VerCINI coverslip with concentrated cell cultures is centrifuged to load cells into holes. (iv) Cells not loaded into holes are rinsed off with fresh media. (d) Assembly of full flow chamber with loaded cells. PDMS $\mu$ VerCINI coverslip is dried around the edges, and all but a thin layer of liquid is left above the loaded cells. The plastic cover of the double-sided tape is removed and the tape is adhered to the PDMS. The final product is a closed chamber through which fluid can be flowed.

cured (solidified) by baking in an oven, and the solid PDMS-covered coverslip is then removed. After fabrication, these coverslips can remain in storage at room temperature for extended periods.

Protocol for $\mu$ VerCINI coverslip fabrication:

1. Make a 10:1 ratio of PDMS elastomer base (Sylgard 184 Elastomer Base; Dow Corning) : curing agent (Sylgard 184 Elastomer Curing Agent; Dow Corning) by mixing 10 g elastomer base and $1 \mathrm{~g}$ curing agent in a glass or plastic vessel, and stir vigorously.

2. Degas the mixture by placing it in a vacuum chamber and pulling vacuum for 15 min. 
3. Pour $\sim 1 \mathrm{~mL}$ on top of the silicon micropillars (Figure 7ai).

4. Place a microscope coverslip ( $22 \times 22 \mathrm{~mm}^{2}$, Thickness No. 1.5; VWR) on top and press down gently but firmly with a marker cap similar device with a flat face (Figure 7aii). It is important to press down with enough force to produce a thin layer of PDMS between the pillars and the coverslip, but not so hard that the pillars may be damaged.

5. Cover the back of the coverslip with some remaining PDMS. This will make it easier to remove some PDMS after baking.

6. Set the silicon wafer with PDMS and coverslip in an oven and bake at $80^{\circ} \mathrm{C}$ for $1-2 \mathrm{hrs}$.

7. Take the wafer out of the oven and peel off the outer layer of PDMS covering and surrounding the coverslip.

8. Use a flat-edged blade, such as the bare blade of a utility knife, to slide under and pull off the PDMS-covered coverslip (Figure 7aiii). Be very careful not to damage the pillars with the blade while doing this.

\section{Microfluidic chamber top assembly}

A fluidic chamber must be assembled that can provide solution exchange over the microholes in a closed chamber. First, the top of the fluidic chamber is made so that the full system can be immediately assembled once cells are loaded into the holes (Figure 7b). The top of the fluidic chamber consists of a microscope slide with drilled holes to allow for inlet and outlet tubing to carry solution in and out, along with a piece of double-sided tape that has been cut to form a flow channel. The double-sided tape serves two functions: it forms a thin $(\sim 100 \mu \mathrm{m})$ flow channel between the microscope slide and the tops of the microholes, and it seals the whole system together. Importantly, not all brands of double-sided tape adhere well to PDMS, and hence can produce leaks when solution is flowed through. We have found that Duck and Club brand double-sided tapes work well, while Scotch, Sellotape, and WHSmith double-sided tapes do not.

In this protocol, the top of the fluidic chamber is assembled. Inlet and outlet holes are drilled into a microscope slide using a power drill with diamond-tipped drill bits. A piece of double-sided tape is cut to form a flow channel and adhered to the slide. Finally, inlet and outlet interfaces are assembled with cut pipette tips and tubing, all epoxied in place to ensure the system will be sealed and not leak.

Protocol for microfluidic chamber top assembly

1. Take a microscope slide and estimate where holes need to be drilled (we commonly use a Gene Frame as a guide). Mark these spots.

2. Pipette a small volume $(\sim 100 \mu \mathrm{L})$ of water on top of the spots to prevent glass dust kicking up during drilling.

3. Using a power drill (Dremel, Dremel 4000) with multi chuck (Dremel, 0.8-3.2 mm keyless chuck) equipped with diamond-tipped drill bits (0.75 mm; Kingsley North, cat. no. 1-0500-100), drill all the way through the glass at the two holes.

4. Clean the slide to remove glass dust and other impurities by sonicating in ethanol for $15 \mathrm{~min}$ and wiping with a tissue.

5. Cut off a piece of double-sided tape (Duck, $38 \mathrm{~mm} \times 5 \mathrm{~m}$ ) roughly the size of the coverslip $(22 \times 22 \mathrm{~mm})$.

6. Using either a laser engraver or a blade, cut a groove in the double-sided tape to form the flow channel. The channel must be long enough to reach the inlet and outlet holes, and wide enough in the centre to accommodate the microhole array. 
7. Adhere the piece of tape to the microscope slide so that the holes line up with the ends of the channel (Figure 7b). Ensure that the tape seals well by pressing around all the edges. Do not remove the plastic covering from the other side of the tape.

8. Take two pipette tips ( $10 \mu \mathrm{L}$; Starlab) and cut them in half with a pair of scissors or blade, keeping the thinnest end. Since later these will be protruding upward in the microscope, you may not be able to lower the condenser to the proper position if they are too long.

9. Cut $\sim 2-3 \mathrm{~mm}$ from the ends of the tips (where they are thinnest). We find this prevents the tips from protruding too far through the drilled holes and interfering with fluid flow.

10. Cut two polyethylene tubes (ID $0.38 \mathrm{~mm}$, OD $1.09 \mathrm{~mm}$; Smiths Medical, cat. no. 800/100/120) for inlet and outlet tubing. We use $24 \mathrm{~cm}$ for the inlet and $67 \mathrm{~cm}$ for the outlet.

11. Mix together $\sim 1 \mathrm{~mL}$ rapid-drying epoxy (Araldite) in a disposable dish.

12. Spread some epoxy around the edges of one end of the inlet tubing, careful not to get any in the tubing itself.

13. Insert the inlet tubing firmly into one of the cut pipette tips (Figure $7 \mathrm{~b}$ ).

14. Spread epoxy around the edges of one end of the outlet tubing.

15. Insert the outlet tubing firmly into the other cut pipette tip.

16. Insert the cut pipette tips into the drilled holes of the microscope slide on the opposite side from the double-sided tape (Figure $7 \mathrm{~b}$ ). Ensure that they do not protrude through the holes.

17. Seal the interface by spreading epoxy between the tip and slide.

18. When dry, slide a needle $(0.45 \mathrm{~mm} \times 10 \mathrm{~mm}$; $B D)$ into the outlet tubing entrance to later be connected to a syringe. To slide this in, be mindful that the needle tip can catch onto the plastic of the tubing. Try to avoid this by bending the tubing slightly away from the tip while sliding it in.

\section{$\mu$ VerCINI coverslip cell loading}

As with the original implementation of VerCINI, cells are loaded into the holes by centrifugation (Figure 7c). However, there are several important aspects of $\mu$ VerCINI that differ. Firstly, PDMS is normally quite hydrophobic, and the surface tension of an aqueous cell culture is high enough that the microholes will end up filled with air bubbles rather than liquid or cells. PDMS is therefore first rendered hydrophilic by treatment with oxygen or air plasma. This treatment only renders the PDMS hydrophilic for a relatively short time (a few hours), as material deeper in the PDMS will eventually migrate to the surface and render it hydrophobic again. Secondly, since PDMS does not retain water like agarose does, the cells are prone to smearing across the PDMS and drying out during centrifugation loading. So, concentrated cell culture is held over the microholes with a silicone gasket, and the entire coverslip is covered to prevent drying.

Finally, after cells have been centrifuged into the holes the chamber must be sealed with the doublesided tape (Figure 7d). However, the tape will not form an adequate seal if liquid is trapped under it. So, after loading cells one must be careful to dry all regions of the PDMS outside the microholes, and also to ensure the liquid level over the microholes is both low enough that it won't spread under the tape while sealing and high enough that the cells will not dehydrate.

Protocol for $\mu$ VerCINI coverslip cell loading:

1. Place the PDMS-coated coverslip in a glass dish.

2. Set in a plasma cleaner (Harrick Plasma, cat. no. PDC-002-CE) and pull vacuum.

3. Treat with air plasma for $3 \mathrm{~min}$ (Figure 7ci).

4. Set the coverslip on a 96-well plate adapter for the centrifuge with a small piece of tissue in between. 
5. Place a $9 \mathrm{~mm}$ diameter silicone gasket (Sigma-Aldrich, cat. no. GBL103240) on top of the PDMS microholes and press down around the edges so the silicone is flush with the PDMS.

6. Concentrate cells $100 x$ by centrifuging $2 \mathrm{~mL}$ of $\mathrm{OD}_{600} \sim 0.4$ cell culture at $17,000 \mathrm{rcf}$ for $1 \mathrm{~min}$ and resuspending in $20 \mu \mathrm{L}$ fresh media.

7. Spot cells onto the holes by pipetting $15 \mu \mathrm{L}$ concentrated cell culture into the gasket (Figure 7cii).

8. Cover the coverslip with a hard plastic bottle cap. Tape the cap down to the plate adapter and place it on the centrifuge rotor. This is to prevent significant amounts of liquid evaporation during centrifugation.

9. Tape another bottle cap on the opposite plate adapter as a weight balance.

10. Centrifuge the cells into the holes for $4 \mathrm{~min}$ at 3,220 rcf (Figure 7ciii).

11. Remove the silicone gasket. Rinse off excess cells from the PDMS surface using media by holding the coverslip upside down over a waste container and gently pipetting 1-2 mL media over the surface (Figure 7civ). The liquid on the PDMS surface should appear clear. If it remains turbid, rinse with more media.

12. Dab with a tissue to dry off the edges of the PDMS. Be careful not to dry off the region near the holes themselves.

13. Pipette a small amount of liquid off of the microhole region of PDMS so there is only a thin layer of liquid remaining. Too much liquid can result in a poorly-sealed chamber as liquid spreads under the double-sided tape, but too little liquid runs the risk of dehydrating the cells.

14. Peel off the plastic covering of the double-sided chamber on the top of the flow chamber (Figure 7d).

15. Press the top of the fluidic chamber to the PDMS, ensuring that the groove in the tape is over the microholes (Figure 7d).

16. Seal the chamber fully by pressing around the edges of the tape.

\section{$\mu$ VerCINI imaging}

Imaging cells with $\mu \mathrm{VerCINI}$ is very similar to that of VerCINI, although there are a few special considerations due to the thickness of the PDMS layer through which imaging will occur. We found that we could not successfully acquire images through the $~ 50 \mu \mathrm{m}$ thick PDMS layer on top of a \#1.5 microscope coverslip with a high N.A. oil immersion 100x TIRF objective. We hypothesize that this is due to the refractive index mismatch between PDMS $(n=1.43)$ and glass $(n=1.52)$. We instead use a silicone immersion oil objective (Nikon, CFI SR HP Plan Apo Lambda S 100XC Sil) since the silicone oil refractive index $(n=1.41)$ is similar to PDMS. The silicone immersion oil objective also has a large working distance $(0.3 \mathrm{~mm})$ which is useful for imaging through a relatively thick PDMS layer.

It is essential to correct for drift during image acquisition in order to obtain high-resolution images. For high-resolution microscopy, this is usually achieved via reflection-based autofocus systems that measure the distance between the objective and coverslip using the reflection of an IR LED off the coverslip surface (e.g. Nikon Perfect Focus System). However, $\mu \mathrm{VerCINI}$ is not compatible with reflection-based autofocus systems. This is because very little reflection occurs off the PDMS/liquid interface as the indices of refraction are too similar ( $\sim 1.43$ and $\sim 1.33$, respectively). One possible solution is to use image-based autofocus methods, (e.g. Micro-Manager's OughtaFocus), however these are not suitable for correcting drift during high speed imaging of protein dynamics. Instead, we use an image-based autofocus system that measures drift using the cross-correlation between images and a reference stack in a separate infra-red brightfield illumination and imaging pathway ${ }^{22}$. An IR LED is used to illuminate the sample $(940 \mathrm{~nm}$; Thorlabs, M940L3) and a second camera used to image the IR brightfield image (IDS, UI-1220LE-M-GL). We developed a custom plugin for Micro-Manager to set 
the reference stack, calculate the cross-correlation maps, and maintain sample focus by closed-loop positioning of the microscope stage ${ }^{12}$. We perform the IR drift correction method on a custom built high resolution microscope, however the apparatus for this method can also be retrofitted onto commercial microscopes ${ }^{22}$. If automated drift correction are not available methods, with practise it is possible for a skilled operator to continuously manually correct for drift during image acquisition. However, this will usually lead to reduced image quality due to increased periods of defocus during data acquisition.

When using flow during imaging, we use a commercial syringe pump (Aladdin-220; World Precision Instruments) operated in "withdraw" mode to pull solution through the chamber. This way a single inlet tubing can be moved from one reservoir to another to perform fluid exchange, and any failure to seal the chamber merely results in pulling air rather than producing messy leaks. The reservoirs are kept inside the microscope incubation box so they remain at the same temperature as the sample, and fluid exchange is done by simply moving the inlet tubing from one inlet to another.

It is also important to note that-unlike VerCINI- $\mu$ VerCINI is often used for 'single-shot' experiments, which limits users to recording a single FoV. For example, if a researcher wants to image the effect of an antibiotic perturbation on protein dynamics inside cells (e.g. Figure 3c), then the experiment can't be repeated on a different FoV with the same slide, as all cells on the slide have already been perturbed. Because of this it is imperative not only to have high loading efficiency, but also to take some time to scan across the slide and find the best possible FoV before beginning.

Protocol for $\mu$ VerCINI imaging:

1. Add a drop of silicone objective oil to objective and position the microhole flow device on top of it.

2. Extrude the outlet tubing from the microscope incubation box and connect the needle to a 20 $\mathrm{mL}$ syringe (BD Plastipak).

3. Place the syringe pump adjacent to the incubation box and connect the syringe to it.

4. Set the pump to withdraw mode and with the following settings: $6^{\prime \prime}$ syringe diameter, $10 \mathrm{~mL} / \mathrm{s}$ flow rate.

5. Set open tubes containing media (the reservoirs) inside the microscope incubation box just next to the moving stage.

6. Insert the inlet tubing into the first reservoir (e.g. blank media).

7. Switch the pump on to pull liquid through the chamber. For initial filling of the chamber it is helpful to use a higher flow rate than will be used during experiments. After filling, change the flow rate to something lower, like $1.1 \mathrm{~mL} / \mathrm{s}$.

8. In Micro-Manager, open the NanoJ Live Drift Correction plugin.

9. Select either $\mathrm{Z}$ correction or $\mathrm{XYZ}$ correction from the combo box.

10. Move to an area away from the microholes and run the background subtraction routine.

11. Run the calibration routine to get the pixels-to-distance conversion. Since the conversion factor is saved, this routine only needs to be done once per microscope.

12. Find your region of interest and position the stage at your desired focal plane.

13. Start the autofocus routine. The $Z$ stage will initially move up and down to take a reference stack, and then begin autofocus/drift correction.

14. When ready to change fluids, simply move the inlet tubing from one reservoir to another. 
We focus here on our most common VerCINI image analysis use case: analysis of protein motion around the cell circumference or cell septum via kymograph analysis. This analysis method is appropriate when protein motion is mostly restricted to the leading edge of the cell septum (e.g. FtsZ) or the cell sidewall (e.g. MreB or other elongasome proteins). For other datasets, such as TIRF imaging of cell pole-localized proteins, other analysis methods such as single molecule tracking may be more appropriate. The overall workflow for image processing and analysis is shown in Figure 8a.

\section{Software installation}

The first steps of image processing are done in Fiji/Image ${ }^{23}$. We have developed a plugin called VerciniAnalysisJ specifically for processing VerCINI videos. VerciniAnalysisJ, along with all dependencies, can be installed from the VerciniAnalysisJ update site.

Later image processing steps such as subtracting the cytoplasmic background signal and producing kymographs are done in MATLAB. We have developed software for these steps in a package called ring-fitting 2 that is publicly available on GitHub.

Protocol for software installation:

1. Install VerciniAnalysisJ for Fiji.

a. Install Fiji from the website: https://fiji.sc.

b. Load Fiji and go to Help>Update...>Manage update sites

c. In the Manage update sites GUI, find and add VerciniAnalysisJ.

d. Close the Manage update sites GUI, and press Apply changes in the ImageJ Updater.

e. Restart Fiji.

f. Changes to the plugin may be made by editing the source code available at https://github.com/HoldenLab/VerciniAnalysisJ.

2. Install ring-fitting2 for MATLAB.

a. Ensure that MATLAB (version 2018b or later) and the MATLAB Image processing toolbox and Optimization toolboxes are installed.

b. Download the ring-fitting2 package from GitHub into a directory on your PC: https://github.com/HoldenLab/ring-fitting2.

c. Open MATLAB and set a path to this directory by clicking 'Set Path' in the Home tab, then 'Add folder', then find the 'ring-fitting2' folder, then click 'Save'. Once this step is complete, the path is set indefinitely and there is no need to repeat this step in future analysis.

\section{Image pre-processing}

We have found that image denoising allows us to substantially reduce illumination intensity while still maintaining high signal to noise ratio, thereby minimizing photobleaching and phototoxicity. We strongly recommend denoising VerCINI data prior to subsequent analysis. While a number of denoising algorithms have been developed, we use the ImageJ plugin PureDenoise, which is based on wavelet decomposition ${ }^{24}$. One advantage to using this algorithm is that it does not make any assumptions about the underlying biological structure.

After denoising, any global image drift-for example due to agarose contraction-is corrected via image registration using the ImageJ plugin StackReg ${ }^{25}$. Trapped cells are identified from fluorescence images (Figure 8c), and cropped images containing images of individual cells are exported for VerCINI image analysis (Figure 8d). Fluorescence movies, together with corresponding short brightfield movies are inspected at this point to exclude cells that are not properly trapped in the microholes. Improperly 
a
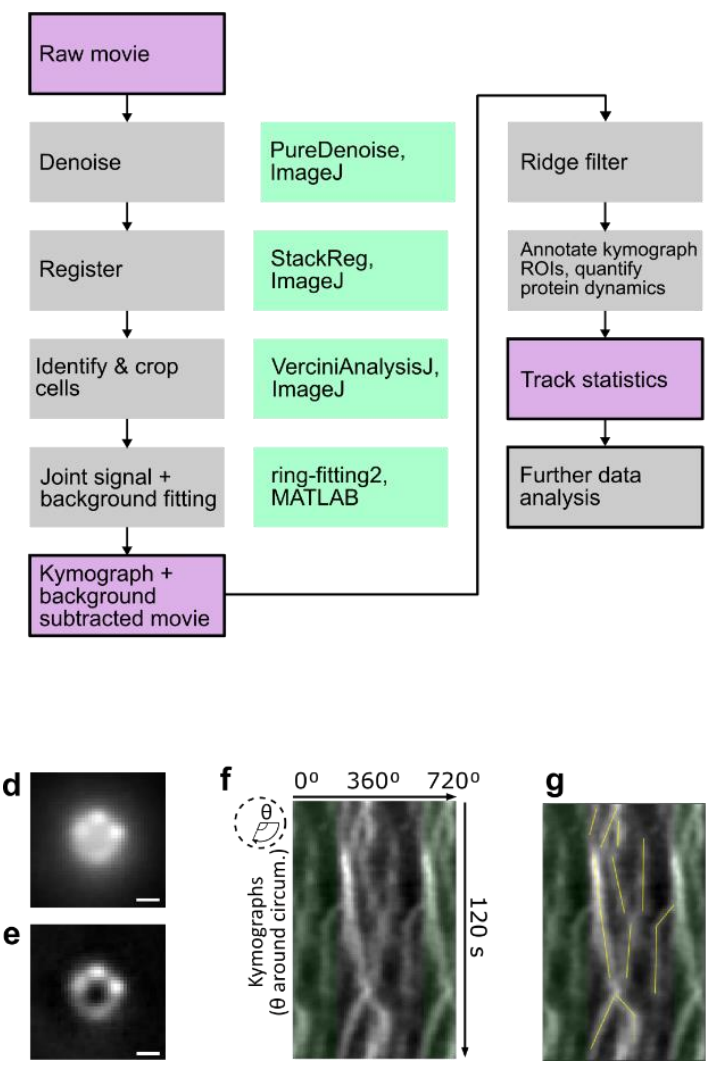

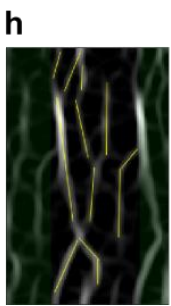

b

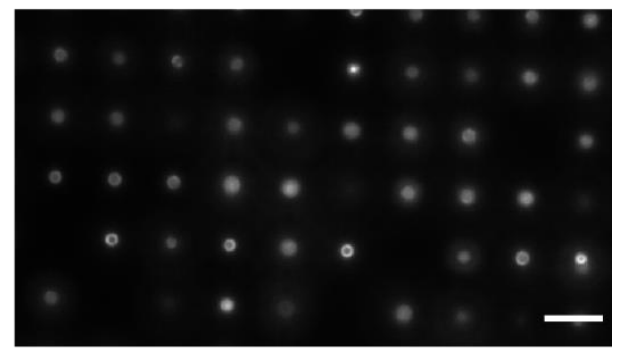

C
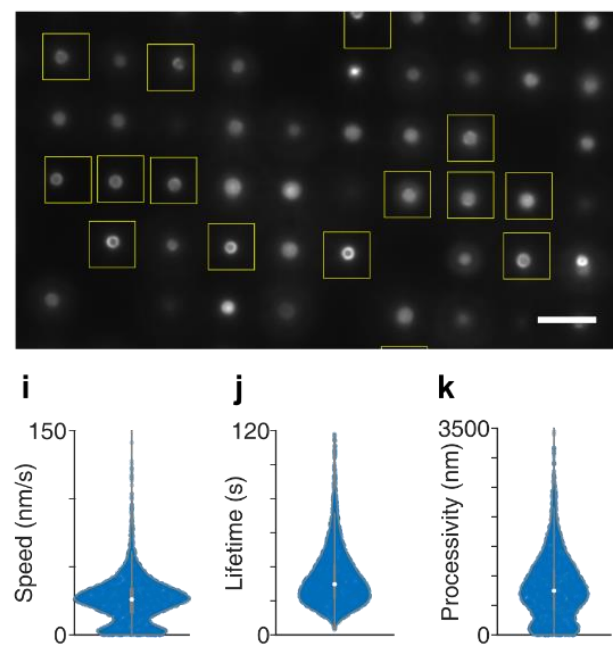

Figure 8: Image processing and analysis for VerCINI and $\mu$ VerCINI. (a) VerCINI image processing workflow diagram. Purple box, data. Grey box, Image/ data processing step. Green box, software tool to perform image/ data processing step. (b) Exemplar VerCINI image of B. subtilis cells expressing FtsZ-GFP. Scale bar, $5 \mu \mathrm{m}$. (c) Regions of interest around in-focus cell septa/ circumference are manually identified and cropped for further analysis. Scale bar, $5 \mu \mathrm{m}$. (d) Exemplar denoised VerCINI image of a single cell. Scale bar, $0.5 \mu \mathrm{m}$. (e) Background subtracted image using joint model-based VerCINI fitting and background subtraction algorithm. (f) Kymograph around septum of background subtracted cell in e. (g-h) Annotated raw kymograph (g) and ridge-filtered kymograph (h). Line ROls indicate manually detected and annotated filament trajectories. Green regions in $f-h$ indicate repeated section of kymograph added to visualise filament trajectories crossing the boundary of the circular profile. (i-k) Exemplar violin plots of FtsZ-GFP filament dynamics measured by VerCINI. Raw data in panels b-k from Whitley, Jukes et al. $(2021)^{12}$.

trapped cells show visible diffusion within the microholes, especially within the brightfield images. If the sample has previously been well characterized, inspection of the fluorescence movies alone may be sufficient.

Protocol for image pre-processing:

1. The following datasets are required or recommended:

a. Fluorescence timelapse of protein dynamics (essential)

b. Brightfield movie recorded after fluorescence timelapse to exclude poorly trapped cells (recommended),

c. Fluorescence Z-stack recorded after fluorescence timelapse to ensure correct Z-plane has been chosen and exclude cells with bright structures immediately above or below imaging plane (sample dependent, recommended for cell division proteins)

2. Open the raw TIF files in ImageJ.

3. Play VerCINI movies and identify cells showing in-focus protein localization that are well trapped. These cells are suitable for subsequent analysis.

4. Go to Plugins > VerciniAnalysisJ > 'Start Vercini ActionBar' 
5. On the ring action bar:

a. Click 'Start ROI manager'

b. Click 'Draw $60 \times 60 \mathrm{ROI}^{\prime}$ - this will draw a square region of interest in the active image.

c. Drag the ROI over each identified cell and use the ' $T$ ' key to record the cell position.

d. Repeat ROI selection and logging step for all identified cells

e. Click 'Save ROls'. This will create an 'roi.zip' file containing the positions of identified cells in the same directory as the TIF file.

f. Repeat the above steps for any other VerCINI movies to be analysed.

g. Click 'Batch denoise+register+crop'. Select the input directory containing the TIF+roi.zip files for analysis.

h. This will produce a denoised and registered '.tif' file and a folder called 'Indiv_rings' containing each individual cell cropped to the selected region of interest. Note: the denoising step may take some time ( $>0.5 \mathrm{hrs}$ ) for large datasets.

\section{Model-based background subtraction and calculation of circumferential kymographs}

A common application of VerCINI is to measure protein dynamics around the cell circumference or cell septum. For septal localized proteins, this confines motion to a 1D circle around the edge of the cell. For cell sidewall proteins, motion is confined to a 2D cylindrical surface which appears as a 1D circle on the microscope camera. In either case, a convenient analysis method is to measure protein dynamics in a polar $(r, \theta)$ coordinate system via kymograph analysis. Kymographs are a type of distance-time graph formed by sequentially plotting $1 \mathrm{D}$ intensity profiles over time to form a 2D image (Figure 8f).

Our ring-fitting 2 software automatically extracts kymographs of circumferentially localised protein dynamics ${ }^{26}$ (Figure $8 \mathrm{~d}$-e). This software also subtracts the diffuse out-of-focus cytoplasmic background (Figure $8 \mathrm{~d}$ ), which can otherwise obscure protein dynamics. The software functions as follows.

Given a VerCINI image stack of a single trapped cell, the circle defining the septal or cell circumference is determined to sub-pixel precision by fitting each image to a joint explicit model for diffuse out-offocus cytoplasmic background (Gaussian + Cauchy model) plus septal/ circumferential protein signal, modelled as a 12 sectored annulus with each sector of variable amplitude ${ }^{12}$ :

$$
F(x, y)=\operatorname{Signal}(x, y)+\operatorname{Bg}(x, y)
$$

Signal fitting is performed in polar coordinates, around the cell centre $\left(x_{0}, y_{0}\right)$, and then converted into Cartesian coordinates after fitting. Signal is defined as

$$
\operatorname{Signal}(r, \theta)=A_{i}(\theta) \exp \left(-\frac{\left(r-R_{0}\right)^{2}}{2 \sigma^{2}}\right),
$$

where $A_{i}(\theta)$ represents the amplitudes of a 12 sectored blurred annulus, $R_{0}$ is the centre of the cell, and $\sigma$ is the standard deviation based on the diffraction limit of light for this system. Background is defined as

$$
\operatorname{Bg}(x, y)=a \exp \left(\frac{-\left(\left(x-x_{0}\right)^{2}+\left(y-y_{0}\right)^{2}\right)}{2 \sigma_{b g 1}^{2}}\right)+b \frac{\sigma_{b g 2}^{2}}{\left(x-x_{0}\right)^{2}+\left(y-y_{0}\right)^{2}+\sigma_{b g 2}^{2}}+c .
$$

where $a$ and $b$ are the amplitude of the Gaussian and Cauchy distributions jointly describing the cytoplasmic background, $c$ is image baseline, $\sigma_{b g 1}$ is the width of the Gaussian distribution, and $\sigma_{b g 2}$ is the width of the Cauchy distribution.

The background signal is subtracted from the image stack for each frame, and then the intensity around the fitted circle is calculated to sub-pixel precision via interpolation. 
We found that sub-pixel fitting of the cell centre and septum/ circumference and robust background subtraction were crucial to obtaining accurate intensity measurements. During software development, we observed that small inaccuracies in cell centre localization, caused for example by fitting an annulus with uniform instead of sectored amplitude would cause large errors in apparent septal intensity as the small size of the cell, together with the complex background meant that different amounts of background signal would be integrated on either side of the cell. In order to confirm that the microscope system and image processing pipeline are together giving even circular symmetric intensity measurements around the centre of the cell, a cell expressing cytoplasmic GFP can be imaged and analysed. An example script for this purpose is supplied in the VerCINI analysis software.

As the VerCINI circle-fitting method is performed on a per-frame basis, the analysis method works equally well on septa that constrict noticeably over the data acquisition period. In this case, analysis of constriction rate can also be performed as the cell radius for each frame is returned as a parameter of the analysis.

Protocol for background subtraction and calculation of circumferential kymographs:

1. Within the 'ring-fitting2' $>$ 'testing' is an example script named 'testVerciniAnalysis. $m$ ' to run with our default settings. Copy and paste this file into the 'Indiv_rings' folder produced by the Fiji 'VerciniAnalysisJ' plugin.

2. Click the 'browse to folder' button and select the 'Indiv_rings' folder

3. In the 'Current Folder' panel, double click the 'testVerciniAnalysis. $m$ ' file and change any options as required, especially the fname variable defining the files you want to analyse.

4. More information about options can be found in the documentation in the GitHub repository. Detailed documentation of all the optional arguments to the VerCINI software may be accessed by typing 'doc verciniAnalysis' or 'doc manualVerciniAnalysis' in MATLAB.

5. In the 'Editor' tab, click 'Run'. Within 'Indiv_rings', a new folder named 'analysed' is created containing the kymograph (with and without background subtraction), and the background subtracted VerCINI movie.

\section{Kymograph analysis of protein dynamics}

Processive protein motion is visible on kymographs as a diagonal line, with line angle indicating protein speed. We currently quantify protein speed, bound lifetime, and other parameters such as directional switching and pausing by manual kymograph annotation (Figure 8g-h), as we found that most automated kymograph annotation methods do not perform well at high density, at least for dense protein filaments such as FtsZ. An ImageJ macro is provided for quantification of filament dynamics via manual ImageJ regions of interest (ROIs) annotation. During kymograph analysis, it can be difficult to identify trajectories of dim filaments due to the large intensity range within kymographs. This issue is frequently encountered for analysis of FtsZ dynamics in dense Z-rings. To address this issue, we recommend applying a ridge detection filter to the kymograph, which detects peaks within an image irrespective of intensity based on the image second derivative. A script 'Ridge_Filter.ijm' is provided for this purpose. We note that a recent deep learning based kymograph annotation tool could enable automated processing of VerCINI kymographs in the future ${ }^{27}$.

Protocol for kymograph analysis:

1. In ImageJ, open 'Vercini ActionBar' and elect 'Start ROI Manager'.

2. Open the '_KymoRawWrap.tif' images to analyse.

3. Optionally, use the Vercini ActionBar 'Ridge Filter' to highlight ridges in the image.

4. For each image, use the straight line tool to trace over the kymograph lines and use the ' $T$ ' key to add the trace to the ROI manager. 
5. On the Vercini ActionBar, select 'save ROIs' to save an roi.zip file for each image.

6. Once all of the kymographs have been traced, select 'Batch kymotrace statistics'.

7. Define the camera pixel size and the frame interval of the images, then select the folder containing the images and roi.zip files.

8. The results are output in a new ImageJ window and can be saved as a .csv file.

\section{Conclusion}

Light microscopy of bacteria provides a wealth of information about their organization, but conventional imaging approaches are limited to viewing bacteria along their long axes only. VerCINI provides a complementary approach by orienting bacteria vertically and imaging along their short axes, thereby vastly improving the imaging of many biologically important structures and dynamic processes in non-spherical bacteria that were previously difficult to observe. VerCINI has already found multiple applications in high resolution imaging of bacterial spatial organization in hands of a small number of early-adopter labs ${ }^{5,12-16}$. We hope that the methods and protocols presented here will allow many other labs to use VerCINI to look at diverse questions in bacterial cell biology from a different angle.

\section{Authorship Contribution Statement}

$\mathrm{KDW}, \mathrm{SM}$, and $\mathrm{CJ}$ performed the experiments. KDW, $\mathrm{SM}, \mathrm{SH}$, and $\mathrm{CD}$ wrote the paper.

\section{Acknowledgements}

We would like to acknowledge Sergii Pud (TU Delft; now at Twente) for help with wafer design and nanofabrication, Siddharth Deshpande (TU Delft; now at Wageningen) for help with nanofabrication and soft lithography, Marc Zuiddam (TU Delft) for help with wafer etching, and Henrik Strahl (Newcastle) for strains and helpful discussions. SH, KDW, CJ, SM acknowledge funding support by a Wellcome Trust \& Royal Society Sir Henry Dale Fellowship [206670/Z/17/Z]. CD acknowledges funding support by from the ERC Advanced Grants 883684 and 669598, and the NanoFront and BaSyC programs. SM supported by a UK Biotechnology and Biological Sciences Research Council doctoral studentship.

\section{Competing Interests}

The authors declare no competing interests.

\section{References}

1. Huang, B., Babcock, H. \& Zhuang, X. Breaking the Diffraction Barrier: Super-Resolution Imaging of Cells. Cell 143, 1047-1058 (2010).

2. Pasquina-Lemonche, L. et al. The architecture of the Gram-positive bacterial cell wall. Nature $582,294-297(2020)$. 
3. Garner, E. C. et al. Coupled, Circumferential Motions of the Cell Wall Synthesis Machinery and MreB Filaments in B. subtilis. Science 333, 222-225 (2011).

4. Domínguez-Escobar, J. et al. Processive Movement of MreB-Associated Cell Wall Biosynthetic Complexes in Bacteria. Science 333, 225-228 (2011).

5. Bisson-Filho, A. W. et al. Treadmilling by FtsZ filaments drives peptidoglycan synthesis and bacterial cell division. Science 355, 739-743 (2017).

6. Yang, X. et al. GTPase activity-coupled treadmilling of the bacterial tubulin FtsZ organizes septal cell wall synthesis. Science 355, 744-747 (2017).

7. Kirkpatrick, C. L. \& Viollier, P. H. Poles Apart: Prokaryotic Polar Organelles and Their Spatial Regulation. Cold Spring Harb. Perspect. Biol. 3, a006809-a006809 (2011).

8. Kuwada, N. J., Traxler, B. \& Wiggins, P. A. Genome-scale quantitative characterization of bacterial protein localization dynamics throughout the cell cycle. Mol. Microbiol. 95, 64-79 (2015).

9. Laloux, G. \& Jacobs-Wagner, C. How do bacteria localize proteins to the cell pole? J. Cell Sci. jcs.138628 (2014) doi:10.1242/jcs.138628.

10. Surovtsev, I. V. \& Jacobs-Wagner, C. Subcellular Organization: A Critical Feature of Bacterial Cell Replication. Cell 172, 1271-1293 (2018).

11. Bowman, G. R., Lyuksyutova, A. I. \& Shapiro, L. Bacterial polarity. Curr. Opin. Cell Biol. 23, 71-77 (2011).

12. Whitley, K. D. et al. FtsZ treadmilling is essential for Z-ring condensation and septal constriction initiation in Bacillus subtilis cell division. Nat. Commun. 12, (2021).

13. Perez, A. J. et al. Movement dynamics of divisome proteins and PBP2x:FtsW in cells of Streptococcus pneumoniae. Proc. Natl. Acad. Sci. 116, 3211-3220 (2019).

14. Söderström, B., Chan, H., Shilling, P. J., Skoglund, U. \& Daley, D. O. Spatial separation of FtsZ and FtsN during cell division. Mol. Microbiol. 107, 387-401 (2018).

15. McCausland, J. W. et al. Treadmilling FtsZ polymers drive the directional movement of sPGsynthesis enzymes via a Brownian ratchet mechanism. Nat. Commun. 12, 609 (2021). 
16. Trouve, J. et al. Nanoscale dynamics of peptidoglycan assembly during the cell cycle of Streptococcus pneumoniae. Curr. Biol. S0960982221005765 (2021) doi:10.1016/j.cub.2021.04.041.

17. Bakshi, S., Choi, H. \& Weisshaar, J. C. The spatial biology of transcription and translation in rapidly growing Escherichia coli. Front. Microbiol. 6, 636 (2015).

18. Strahl, H. et al. Transmembrane protein sorting driven by membrane curvature. Nat. Commun. 6, (2015).

19. Briegel, A. et al. New Insights into Bacterial Chemoreceptor Array Structure and Assembly from Electron Cryotomography. Biochemistry 53, 1575-1585 (2014).

20. Chai, Y., Norman, T., Kolter, R. \& Losick, R. An epigenetic switch governing daughter cell separation in Bacillus subtilis. Genes Dev. 24, 754-765 (2010).

21. Ellefsen, K. L., Dynes, J. L. \& Parker, I. Spinning-Spot Shadowless TIRF Microscopy. PLOS ONE 10, e0136055 (2015)

22. McGorty, R., Kamiyama, D. \& Huang, B. Active microscope stabilization in three dimensions using image correlation. Opt. Nanoscopy 2, 3 (2013).

23. Schindelin, J. et al. Fiji: An open-source platform for biological-image analysis. Nat. Methods $\mathbf{9}$, 676-682 (2012).

24. Luisier, F., Vonesch, C., Blu, T. \& Unser, M. Fast interscale wavelet denoising of Poissoncorrupted images. Signal Process. 90, 415-427 (2010).

25. Thevenaz, P., Ruttimann, U. E. \& Unser, M. A pyramid approach to subpixel registration based on intensity. IEEE Trans. Image Process. 7, 27-41 (1998).

26. Whitley, K. D. et al. FtsZ treadmilling is essential for Z-ring condensation and septal constriction initiation in Bacillus subtilis cell division. (Zenodo, 2021). doi:10.5281/ZENODO.4570259.

27. Jakobs, M. A., Dimitracopoulos, A. \& Franze, K. KymoButler, a deep learning software for automated kymograph analysis. eLife 8, e42288 (2019). 
28. Bisson-Filho, A. W. et al. Erratum for the Report: "Treadmilling by FtsZ filaments drives peptidoglycan synthesis and bacterial cell division". Science 367, (2020).

29. Tinevez, J.-Y. et al. TrackMate: An open and extensible platform for single-particle tracking. Methods 115, 80-90 (2017). 